\title{
TeleGate: Multi-user Semi-teleportation for Remote Collaboration in Mixed Reality 360-Videos
}

by

Hyejin Kim

A thesis

submitted to the Victoria University of Wellington in fulfilment of the requirements for the degree of Master of Science in Computer Graphics.

Victoria University of Wellington 2021 



\begin{abstract}
Telepresence systems enable people to feel present in a remote space while their bodies remain in their local space. To enhance telepresence, the remote environment needs to be captured and visualised in an immersive way. For instance, 360-degree videos (360-videos) shown on head-mounted displays (HMDs) provide high fidelity telepresence in a remote place. Mixed reality (MR) in 360-videos enables interactions with virtual objects blended in the captured remote environment while it allows telepresence only for a single user wearing HMD. For this reason, it has limitations when multiple users want to experience telepresence together and naturally collaborate within a teleported space.

This thesis presents TeleGate, a novel multi-user teleportation platform for remote collaboration in a MR space. TeleGate provides "semiteleportation" into the MR space using large-scale displays, acting as a bridge between the local physical communication space and the remote collaboration space created by MR with captured 360-videos. Our proposed platform enables multi-user semi-teleportation to perform collaborative tasks in the remote MR collaboration (MRC) space while allowing for natural communication between collaborators in the same local physical space.

We implemented a working prototype of TeleGate and then conducted a user study to evaluate our concept of semi-teleportation. We measured the spatial presence, social presence while participants performed remote collaborative tasks in the MRC space. Additionally, we also explored the different control mechanisms within the platform in the remote MR collaboration scenario. In conclusion, TeleGate enabled multiple co-located
\end{abstract}


users to semi-teleport together using large-scale displays for remote collaboration in MR 360-videos. 


\section{Acknowledgments}

I would like to express my deepest gratitude to my supervisors, Prof. Taehyun Rhee and Dr. Daniel Medeiros, who provided me with great guidance and continuous encouragement. I am grateful to Prof. Taehyun Rhee for allowing me to undertake this research and his support from the beginning of my stay and throughout my whole study. I appreciate Daniel, who is always available for discussion and offered me invaluable feedback in all the stages. I am thankful that this work is supported by the CMIC Master Scholarship from Entrepreneur University Programme by TEC.

I would like to thank all the academic and research staffs of CMIC, especially to the UXR team for their help and meaningful discussions. Particularly, thanks to Stephen for helping me whenever I ran into a spot of trouble and Dr. Rafael for his encouragement and being a good mentor. Thank you, Dr. Nadia, for providing me sound advice on the user study and always being open to share your expertise.

I wish to express my sincere appreciation to Prof. Daseong Han, Prof. Joseph S. Shin, and CG Lab friends at Handong Global University. Without their help, I would have never been able to start my study in computer graphics.

I am indebted to my parents for their unconditional love and unfailing support over distance, day and night. I love you forever.

Finally, most sincere to my God for giving me the strength and blessing me with these wonderful people around me. Thank you for everything. 


\section{List of Papers}

- F. Welsford-Ackroyd, A. Chalmers, R. K. d. Anjos, D. Medeiros, H. Kim and T. Rhee, "Asymmetric Interaction between HMD Wearers and Spectators with a Large Display," 2020 IEEE Conference on Virtual Reality and 3D User Interfaces Abstracts and Workshops (VRW), Atlanta, GA, USA, 2020, pp. 670-671.

- H Kim, J Young, D Medeiros, S Thompson, T James Rhee, "TeleGate: Immersive Multi-User Collaboration for Mixed Reality $360^{\circ}$ Video", IEEE Symposium on Virtual Reality and 3D User Interfaces 2021 Poster, March 2021 


\section{Contents}

\begin{tabular}{lll}
\hline & Introduction & 1
\end{tabular}

1.1 Motivation . . . . . . . . . . . . . . . . . . . . 1

1.2 Research Objective . . . . . . . . . . . . . . 4

1.3 Contribution . . . . . . . . . . . . . . . 6

1.4 Thesis Structure $\ldots \ldots \ldots \ldots$. . . . . . . . . . . 7

$\begin{array}{lll}2 & \text { Related Work } & 9\end{array}$

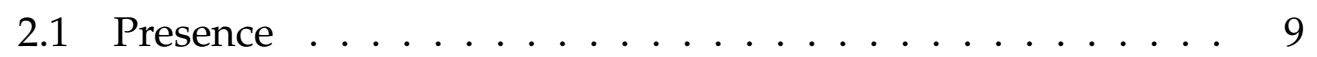

2.2 Collaboration and Communication . . . . . . . . . . . 12

2.3 Co-Located Multi-User Virtual Environment . . . . . . . . . 13

2.4 Remote Multi-User Virtual Environment. . . . . . . . . . . . 16

2.5 Chapter Summary . . . . . . . . . . . . . . . . . . . . . 18

$\begin{array}{lll}3 & \text { TeleGate } & 19\end{array}$

$3.1 \quad$ Concept Overview . . . . . . . . . . . . . . . . . . . . 19

3.2 System Overview . . . . . . . . . . . . . . . . . 22

3.3 System Implementation . . . . . . . . . . . . . . . . . . 25

3.3 .1 Controller Design . . . . . . . . . . . . . . 25

3.3 .2 Camera Control . . . . . . . . . . . . . . . . . 27

3.3 .3 Object Control . . . . . . . . . . . . . . . 30

3.4 Chapter Summary . . . . . . . . . . . . . . . . 35

vii 
\begin{tabular}{|ll}
4 & User Study
\end{tabular}

4.1 Research Question . . . . . . . . . . . . . . . . 37

4.2 Evaluation Design . . . . . . . . . . . . . . . . . . 39

$4.2 .1 \quad$ Experimental Design . . . . . . . . . . . . . . 39

4.2 .2 Task Design . . . . . . . . . . . . . . . . 40

4.2 .3 User Interface . . . . . . . . . . . . . . . . . 41

$4.2 .4 \quad$ Setup and Apparatus . . . . . . . . . . . . . . . . 43

4.3 Materials . . . . . . . . . . . . . . . . . . . . . 44

4.3 .1 Spatial Presence . . . . . . . . . . . . . . 44

4.3 .2 Social Presence . . . . . . . . . . . . . . . . 45

4.3 .3 User preferences . . . . . . . . . . . . . . 46

4.3 .4 Task Performance . . . . . . . . . . . . . . . 47

4.4 Procedure . . . . . . . . . . . . . . . . . . . 47

4.5 Data collection and Analysis . . . . . . . . . . . . . . . 48

4.6 Result and Analysis . . . . . . . . . . . . . . . . . . . . 49

$4.6 .1 \quad$ Demographics . . . . . . . . . . . . . . . 49

4.6 .2 Subjective Responses . . . . . . . . . . . . . . . 49

4.6 .3 Task Performance . . . . . . . . . . . . . . . 57

4.7 Chapter Summary . . . . . . . . . . . . . . . . . . . 60

$\begin{array}{lll}5 & \text { Discussion } & 61\end{array}$

5.1 Presence . . . . . . . . . . . . . . . . . 61

$5.1 .1 \quad$ Spatial Presence . . . . . . . . . . . . . . . . . 61

5.1 .2 Social Presence . . . . . . . . . . . . . . 62

5.2 User preferences . . . . . . . . . . . . . . . . . . . . 64

5.3 Task Performance . . . . . . . . . . . . . . . . . . . 66

5.4 Suggested System Improvements . . . . . . . . . . . . . 66

5.5 Application . . . . . . . . . . . . . . . . 67

5.6 Chapter Summary . . . . . . . . . . . . . . . . . . 68

$\begin{array}{llr}6 & \text { Conclusion } & 69\end{array}$

6.1 Limitation and Future Work . . . . . . . . . . . . . . 70 
6.2 Chapter Summary . . . . . . . . . . . . . . . . 72 


\section{List of Figures}

3.1 Overview of the gate portal concept. . . . . . . . . . . 20

3.2 Left: Large-scale video-wall displays to act as a gate portal between the local space and the remote space. Right: 6DoF motion controllers to capture the users' movement for changing viewpoints and manipulating objects. . . . . . . 22

3.3 Overview of the TeleGate working prototype. . . . . . . . . 24

3.4 Manipulating incorporates four buttons: grip button, trigger, pad button, menu button. . . . . . . . . . . . . 26

3.5 Two Vive trackers placed on each lower corner of the displays to track the displays in the real world for creating a virtual screen and positioning the virtual camera. . . . . . . 27

$3.6 \quad$ Discordance of the position between the camera and each

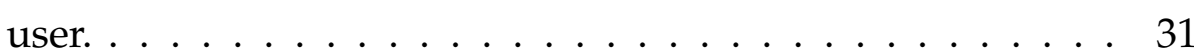

3.7 Explanation of the correction performed on a user's pointing ray. To enable the corrected ray's perspective from the user's viewpoint, first we perform a ray-casting between the controller's forward ray and the screen (r1), then we project this point into the camera (r2), where we perform the ray-casting using the corrected perspective $(\mathrm{r})$. . . . . . 32

$4.1 \quad$ Three different control mechanisms between each group of users enabling different collaboration strategies. . . . . . . 39 
4.2 Example of the reference paper given to each group of participants which indicates five target objects. . . . . . . . . . 40

$4.3 \quad$ 2D user interface at the top of the large-scale displays to show each participant's state of the controller. . . . . . . . . 41

4.4 3D virtual indicators as a reference to support participants. Left: A 3D arrow pointing to where a target object should be

\begin{tabular}{|c|}
\hline relocated. Right: A ghost object indicating correct rotation \\
\hline
\end{tabular}
and scale. . . . . . . . . . . . . . . . . . . . 42

4.5 Three participants conducting the collaborative task together. 43

4.6 Spatial presence results between different control mechanisms. Green represents the local space, and orange represents the MRC space. . . . . . . . . . . . . . . . 51

4.7 Social presence results between different control mechanisms. Green represents the local space, and orange represents the MRC space. . . . . . . . . . . . . . 53

4.8 User preferences results analysed by four metrics. Pink represents Solitary control (A), yellow represents Identical control (B), and sky blue represents Limited control (C). . . . . . 57

4.9 Boxplots representing the completion time per trial in seconds. . . . . . . . . . . . . . . . . 59 


\section{List of Tables}

$4.1 \quad$ Spatial Presence Questionnaire . . . . . . . . . . . . . . . 44

4.2 Social Presence Questionnaire . . . . . . . . . . . . . . . 45

4.3 User Preferences Questionnaire . . . . . . . . . . . . . . . . 46

4.4 Overall subjective rating results on 7-point Likert scale presented as Median (Interquartile range). AA (Attention Allocation), SSM (Spatial Situation Model), SPSL (Spatial Presence Self-Location), SPPA (Spatial Presence Possible Actions), IIP (Impression of Interaction Possibilities). . . . . . . . . . 50

4.5 The presence result of Wilcoxon Signed Ranks Test $(a=$ $0.05)$ as post-hoc tests between local space and MRC space in each control mechanism. Star $\left(^{*}\right)$ indicates statistical significance. . . . . . . . . . . . . . . . 55

4.6 The presence result of Friedman parametric tests with Wilcoxon

\begin{tabular}{|c|}
\hline Signed Ranks Test $(a=0.05)$ as post-hoc tests between rat- \\
\hline \hline ing scores of presence and the neutral line in each control \\
\hline \hline mechanism. The Bonferroni correction (sig. $=$ sig. $/ 3$ ) was \\
\hline \hline applied. Star $\left(^{*}\right)$ indicates statistical significance. . . . . . . 56
\end{tabular}


4.7 Top: (A) Solitary control, (B) Identical control, (c) Limited control. The user presences result of Wilcoxon Signed Ranks Test $(a=0.05)$ as post-hoc tests between different control mechanisms as a pairwise comparison. Bottom: The user presences result of Wilcoxon Signed Ranks Test $(a=0.05)$ as post-hoc tests between rating scores of user preferences and the neutral line in each control mechanism. Bonferroni correction (sig. $=$ sig. $/ 3$ ) was applied. Star $\left(^{*}\right)$ indicates statistical significance. . . . . . . . . . . . . . 58 


\section{Chapter 1}

\section{Introduction}

In this chapter, we introduce the motivation of our research by some limitations that the current telepresence systems have faced in terms of multiple users. Then, we propose how our approach can solve existing problems through our two research objectives. Finally, we summarise the contributions we made and describe the flow of thesis content with a brief explanation.

\subsection{Motivation}

Technologies have been fostering to connect people to communicate with anyone, anywhere, and anytime. As the network improved, it is even possible for people to collaborate at great distances without losing time travelling [37]. Particularly, immersive technologies show great promise enabling people to work together through the network across great distances irrespective of their locations.

Virtual reality (VR) describes a 3D computer-generated environment which can be explored and interacted with by a person who becomes part of this virtual world or is immersed in this environment. In contrast, Augmented reality (AR), which allows virtual objects generated by computer graphics to be overlaid into the physical world in real-time, facilitates a 
user to see the augmented real world, rather than completely replacing it like VR. Mixed reality (MR), a hybrid of VR and AR, which empowers real-world and virtual objects by putting them together, produces a new environment where physical and virtual objects interact.

Telepresence systems have been enhanced in recent years by the use of MR technology, which allows people to feel connected to both a virtual environment and their remote peers. The environment needs to be fully recreated with a high level of fidelity, i.e., similar to the way it actually is in real life [28]. This is especially important in situations where contextual information is required to provide a rich understanding of the environment in order for people to communicate and interact with both other users and the environment itself. However, this can be especially challenging for the virtual environment that recreates real physical spaces using depthcameras, as it may limit the sense of the presence of the user [62].

Current 360-degree capturing cameras (360-cameras) and videos (360videos) allow live capturing, streaming, and visualisation of the omnidirectional view of the surrounding real environment. 360-videos shown in HMDs allow for a high sense of telepresence [39, 62], and MR in 360videos enables user interactions in the captured environment [48, 52, 62]. Recently, augmented virtual teleportation (AVT) [53] presented a platform for remote collaboration in MR using a 360-camera as a teleportal. A remote VR traveller using an HMD collaborates with an AR host in a MR collaboration space while eliciting a high sense of spatial presence and co-presence in the captured environment as if they were in the same collaboration space.

Still, previous research has focused on allowing only one traveller to teleport and did not consider multiple travellers to collaborate with together. However, in specific scenarios where complex problems involving many experts, such as civil engineering, consultation, and training, multiple users can benefit from immediate communication and perform better [33]. There are only a few existing approaches found in the litera- 
ture which enable groups of people to explore together (or teleport to) the virtual space [33, 43]. Existing systems are still limited in terms of their representation of low-fidelity versions of people [5] or their lack of representation of the remote site where the collaboration takes place and restrict interaction to a single-user in the co-located space [5, 43].

For multiple users to be able to collaborate in the same space, they need to be able to naturally communicate with each other while maintaining a high sense of presence in the virtual space. Augmented 360-videos often allow for a high degree of visibility, but they also have a fixed viewpoint of the reconstructed scene utilising 360-videos. In order to ensure that the scene is not distorted, users need to be positioned at the same place where the scene was captured. This becomes problematic when multiple users simultaneously experience the virtual environment, which limits non-verbal communication between users and compromising the overall immersive experience.

Furthermore, most approaches found in the literature are dependent on head-mounted displays (HMDs) [21, 52, 53] that provide a complete immersion experience that shuts out the physical world. As HMD obscures each user's face, it provides a high level of presence. However, since it requires people's real bodies to be represented inside the virtual environment in forms of avatars losing natural non-verbal cue, it becomes difficult for them to understand each other's non-verbal references such as eye contact.

To overcome these issues, co-located multiple users need to be provided communicable space where they are free from HMDs while keeping a sense of presence in the teleported space. Large-scale displays can be an alternative to HMDs as they still enable a high sense of presence and allow multiple users to work together in the same physical space without the need for them to be reconstructed as 3D avatars in the virtual environment. Further, they cause fewer side-effects [54], while maintaining a similar level of spatial awareness when compared to HMDs [45]. 
In the next section, we propose our setup to solve these problems by suggesting our research objectives.

\subsection{Research Objective}

The research objective is to implement a teleportation system that enables co-located multiple users to collaborate in remote space while keeping natural communication in their local space. To achieve this, we present the two main objectives of our research.

The first objective is to provide a communication space in local space to enhance face-to-face communication. In most co-located collaboration scenarios in VE, all users need to wear their own HMD. As HMDs cover each user's face, it makes users feel distanced from their collaborators and ineffectively support nonverbal cue such as eye contact that happen in normal face-to-face communication. The removal of HMDs can enable multiple users in the same space to see their collaborators easily and communicate. Therefore, by using large-scale displays instead of HMDs, multiple users can position side-by-side around the displays to engage in collaboration while still having a presence in VE.

The second objective is to create a shared remote workspace to manipulate virtual objects for conducting collaborative works. Through the large-scale displays, users share the view of the remote space and are aware of remote real-world surroundings for spatial information. In addition to this, we go beyond previous work on large-scale displays that limited the interaction to a single person at a time, by allowing every user to control their view and interact with virtual objects with their control ability.

Our approach aims to preserve the natural interaction between multiple users to perform tasks together in the remote workspace. At the same time, it enables users to feel a sense of spatial presence which is the illusion of "being there" [30] in the teleported remote space and social pres- 
ence which is the feeling of "being together" [58] with other collaborators. To evaluate our approach, a user study was conducted measuring users' presence and preferences when using our platform for collaboration.

In the next section, we summarise how we contributed to achieve these research objectives by our implementation. 


\subsection{Contribution}

The main contributions of our research are summarised as follows:

- We present TeleGate, a novel multi-user teleportation platform for collaboration in the the remote workspace. It enables semi-transportation that allows multiple users to collaborate in the MR collaboration (MRC) space while preserving natural communication among multiteleporters within their local physical space.

- We implemented a working prototype of the TeleGate using a commercial game engine (Unity 3D), which provides immersive and interactive interfaces for multi-user MR collaboration. Users can interactively manipulate virtual objects seamlessly blended into 360videos while sharing the view in large-scale displays.

- We conducted a user study to evaluate semi-teleportation using the TeleGate. We measured the spatial and social presence in both the local physical space and the remote MRC space. We also evaluated user preferences with different control mechanisms for accomplishing collaborative manipulation tasks.

To the best of our knowledge, none of the existing MR systems have leveraged a form of a shared display for multiple users to communicate while seeing each other and having an influence on the remote teleported space. We expect that our platform has the potential to be applied in various industries requiring co-located users to collaborate together. 


\subsection{Thesis Structure}

This thesis is organised as follows:

- Chapter 2 reviews key works related to our research objectives. This chapter explains the concept of presence and collaborative works and introduces collaborative virtual environments (CVEs) regarding co-located and remote multi-user cases. Then, we address our solution to tackle the existing challenges.

- Chapter 3 describes the concept overview and the system overview of our proposed approach. This chapter also illustrates how our platform was implemented covering camera control and object control for users to collaborate in remote space.

- Chapter 4 presents how user study was designed and carried out to evaluate our platform. This chapter begins with our research question for the user study and then addresses our evaluation design, the methodology, and the result and a brief analysis.

- Chapter 5 discusses our interpretation of the user study based on the analysis of the results obtained from chapter 4 . Then it describes suggested system improvements and applies the insight gained to the related research area.

- Chapter 6 demonstrates the limitations and future works of our research. Finally, this chapter summarises the contributions and conclusions of this thesis. 


\section{Chapter 2}

\section{Related Work}

In this chapter, we review key related work on collaborative virtual environments (CVEs) for multiple users. We start the review by introducing the concept of presence and collaborative works in a virtual environment (VE). We then discuss some important topics related to CVEs for both colocated and remote multi-user collaboration. Finally, we highlight our research contribution that supports for co-located multiple users to teleport together, enabling collaboration in the remote space.

\subsection{Presence}

The concept of presence is defined variously as it describes the subjective experience of a user towards mediated environments [37]. Presence plays a significant role in a virtual environment (VE), being related to how observers vividly experience their surroundings in the virtual environment, and psychologically this implies that users experience the virtual object as an actual object [25, 40]. Presence can be further divided into two subcategories: spatial presence and social presence.

Spatial presence implies the feeling of being present in the mediated environment rather than at the real physical location [29, 59, 66]. It gives observers a sense of feeling that they are actually at the remote site [55], 
as if they have been transported there. Putting this together, spatial presence is the illusion of "being there" [30], thereby enabling to forget the real world. Spatial presence, referred to as "telepresence" in a virtual environment, take place when the users are fully immersed, so that they are able to experience vivid spatial illusions [40, 67]. Wirth et al. [69] explained the formation of spatial presence experiences by integrating psychological concepts; they argue that spatial presence is formed by two elements: self-location (SPSL) and possible actions (SPPA). Self-location means the impression of being located in the virtual environment, and when one can take action such as moving objects in there, these actions are referred to as possible actions. Adding to this, attention allocation (AA) affects the formation of spatial presence, which suggest that only users who pay attention to the mediated environment would feel presence. Vorderer et al. [63] constructed a verbal questionnaire to measure spatial presence in the context of different types of media and cultures based on Wirth's two-level process model [69].

Social Presence was first conceptualised by Short et al. [56]; this occurs during a mediated conversation in an interpersonal relationship, where one has the feeling of having access to the others' thoughts and emotions [12]. In general, social presence in a virtual environment can be represented as co-presence, which is the psychological sensation of "being together" [58]. It refers to the feeling of being in the same environment as others as part of a collaboration group, regardless of their physical space [13, 72]. Co-presence also refers to the sense of being collocated with virtual agents [30]. Social presence arises when users feel the presence of another individual as a form of behaviour or sensory experience [72]. Frank et al. [14] defined social presence as multi-dimensions composed of co-presence, psychological involvement, and behavioural engagement. According to Frank et al. [14], psychological involvement is associated with an observer's attention to others' emotional states, and behavioural engagement involves a belief that one's actions are connected to others' 
actions. Previous studies have shown that social presence is crucial in the virtual environment, as it is associated with positive communication outcomes [44]. Kraut et al. [32] proved that a greater sense of social presence resulted in higher user task satisfaction and better task performance.

Fontaine [24] considered presence to be a matter of focus, while Witmer [70] focused on how users and the virtual environment are connected, suggesting that involvement and immersion are the two main factors accentuating presence. According to Witmer [70], if users find head-mounted displays (HMDs) uncomfortable, their involvement in the virtual environment would be diminished, as would presence. In addition, control and interaction that closely mimicked the real-world experience and the possibility of navigating within the virtual environment reportedly increased presence [41]. In terms of display characteristics, higher frame rates and higher field of view (FoV) were associated with higher self-reported presence [42, 50]. Even though presence can be measured by objective measures such as bio-markers, behavioural measures, and task performance, subjective measures have been a common approach, obtained by using questionnaires after exposure to a virtual environment [66]. 


\subsection{Collaboration and Communication}

Collaboration involves more than one person working together to achieve a common goal. Advanced technology has been fostering good quality in the working environment for collaboration. The emergence of collaborative virtual environments (CVEs) enables users to collaborate even when being at remote locations. Critical characteristics of collaborative work include the use of multiple viewpoints, context sharing, awareness of others, negotiation and communication [18].

In general, CVEs are realised through the use of 3D display systems [71] such as head-mounted displays (HMDs), multiple 3D TVs or 3D projectors, and robots equipped with 3D displays, and by the use of physical controllers for manipulating virtual content to enhance collaboration [10]. Considerations as to which visualisation device is best suited usually depend on elements such as type of task, cybersickness, and subjective feeling of connectedness between the users and the environment also called presence. In the specific case of CVEs, another aspect that needs to be considered is how much social presence it elicits [56], which involves how well users are able to feel the presence of and interact with others.

Sharing a space with other collaborators facilitates communication and the shared virtual space provides a natural manner of interacting with others [7]. Collaborative spaces can be of two types: communication space and workspace (task space). Communication space refers to users being located where they can communicate using speech, gesture, gaze, and nonverbal cues. The workspace is a shared physical space between collaborators, where the actual task is performed. Shared visual workspace is essential, as it facilitates conversational grounding between users [26]. These spaces can be combined or separated. When collaborators gather around a table, for example, their communication space and workspace are combined, as they use the same medium to communicate and act on the environment. This is commonly the case in collaborative settings us- 
ing virtual reality (VR) technology, as see-through HMDs aim to combine communication space and workspace at once. On the other hand, when using large-scale displays for co-located collaborative work, users stay physically in their communication space while performing actions in the displays, which can be referred to as workspace.

\subsection{Co-Located Multi-User Virtual Environment}

Collaborative virtual environments (CVEs) enable collaboration both in the same physical space and in remote sites using virtual reality (VR) and augmented reality (AR) devices, such as head-mounted displays (HMDs). In the case of co-located collaboration, multiple users can better perform a task in a virtual environment compared to a single user, as they benefit from immediate communication [33]. Even if a helper from a distant place can participate in collaboration, a user performs more efficiently with a co-located collaborator staying side-by-side than as a remote one sharing the physical workspace [26].

However, it is challenging for current CVEs to appropriately support multiple users in the same local space with natural viewpoint and communication. In most cases, all users must wear HMDs that serves as a gate to the virtual world for collaboration [21]. HMDs are typically sold with motion controllers which can provide six degrees of freedom (6-DoF), meaning the controller's rotation and position are both tracked along three axes. This enables each of the users to have a singular viewpoint, enabling them to interact and communicate with each other as if they were in the same room, through the use of an avatar [36]. VR HMDs primarily isolate users entirely from their physical surroundings, including others in the same room, limiting natural face-to-face communication. As natural communication supported by non-verbal cues enhances co-located collaboration, VR HMDs are ineffective in supporting that [4, 23].

For this reason, see-through HMDs were preferred to VR HMDs by 
users in co-located collaboration. See-through HMDs allow users to be able to see each other's non-verbal cues, which led to better performance on some tasks [11]. As such, the Studierstube project [60] presented an augmented collaborative system with co-located multiple users wearing see-through HMDs to manipulate virtual models while seeing each other in the real world, promoting natural face-to-face communication. Nonetheless, each display is required to be connected via networking and wearing HMDs continuously for a long time might be bothersome.

On the other hand, the advantage of large-scale displays is the ability of multiple users to be immersed (or teleported) in the virtual scene, since the surface size of the displays exceeds the user's visual field of view (FoV) [51]. Unlike HMDs, large-scale displays enable people to be physically side-by-side and natural communication without the need for users to be represented by an avatar. These avatars are often abstract and do not capture the expressive capabilities of human representation correctly, limiting collaboration. This is a critical factor for enabling people to be more efficient in collaborative scenarios, so that there can be multiple users at the same time.

For this to happen, virtual elements need to be correctly rendered to allow multiple users to interact with large-scale displays. One possible way is by using stereoscopic displays such as a Cave automated virtual environment (CAVE) system [17, 20, 22]. These large-scale displays systems consist of multiple screens that surround the users, and multiple users can gain a stereoscopic view by wearing three-dimensional shutter glasses. Still, these setups are often limited to a single user, as only one user at a time can correctly perceive the world in three dimensions. This restricts the feeling of presence, thereby affecting interaction and collaboration [8]. The C1x6 system [3, 33] overcame this limitation by using a custom projection setup that enables six people to perceive the world in three dimensions, and can be extended to an immersive group-to-group system [6, 34]. Nevertheless, the setup is not commercially available, and 
it is challenging to implement for members of the general public who do not have technical access and know-how.

Because of that, non-stereoscopic video-tiled displays remain a good alternative to collaboration, as they can be placed around the users, expanding their field of view and their spatial awareness [46]. Their use also enables interacting and manipulating 3D objects with indirect techniques such as ray casting using dedicated devices or point gestures [15].

Additionally, this type of setup requires motion controllers to manipulate objects [15] and control the camera [64]. To support natural interaction, pointing rays are required to be rendered perspectively correct for each user [57]. Thus, interaction with large-scale displays needs to be correctly designed to manipulate objects using techniques such as raycasting and point gestures [15]. 


\subsection{Remote Multi-User Virtual Environment}

Collaborative virtual environments (CVEs) have been supporting not only a co-located situation but also remote collaboration between geographically separated collaborators. In general, remote collaboration is achieved by recognising the environment around the local user for the remote collaborator by capturing the scene in real time. When designing CVEs, representational fidelity of the people and the environment being reproduced needs to be considered. This is particularly important in telepresence systems, where nonverbal communication cues are key to enabling presence. These systems provide teleportation capabilities, where people feel that they are actually being transported to the environment being rendered. One option is the use of depth cameras that generate 3D images which can be rendered and perceived in 3D by the users [25]. However, these types of setups do not yet provide enough fidelity for teleportation.

The use of 360-videos, on the other hand, enables the environment to be correctly perceived and a high sense of presence to be achieved [52]. As 360-cameras effectively offers omnidirectional background, it has been increasingly leveraged to share the surrounding environment of the local scene where the camera is located. One example of this is Hoppin' World [2], which is a commercial VR application that allows multiple users wearing HMDs to explore 360-videos together while communicating each other's avatars in the recorded video only. Another recent example is the Augmented Virtual Teleportation (AVT) [53] that uses a 360-camera as a portal to teleport an immersed user into a remote location in real time, where the one can collaborate with a local collaborator using augmented virtual objects that match the environment light conditions. This setup provides a high sense of presence and co-presence to both remote and local users, though at the cost of limited mobility for the remote user due to 360-cameras limitations. Still, this system is yet to provide the opportunity for more than one remote user to be teleported to participate in the 
collaboration.

An extant body of relevant work has gone further and explored collaboration between groups of users in remote locations. In this context, there are existing works that use both VR and large-scale displays to enable group-to-group collaboration [36, 43]. In both cases, the users are represented by abstract avatars to enable collaboration. Beck et al. [5] went a step further and used a point-cloud representation in conjunction with a large-scale display to represent groups of people in a remote meeting.

Furthermore, researchers have explored promotion of co-presence during a remote collaboration by providing visual information in shared visual spaces for enhanced collaboration [9, 27]. A pointer is one of the effective communication cues, when both collaborators share the same information to guide a task [31]. In the case of Mini-me [47], a remote helper represented as an avatar in a virtual environment instructed the local user to organise a physical object using eye-gaze and body gestures with a pointing ray. Similarly, CoVAR [48] converted a remote user's head-gaze, eye-gaze, and hand gestures into a visual cue to help a local user with manipulating objects of interest, and 3D Helping hands [61] used hand gestures of the remote helper as visual aids in addition to audio communication. Lastly, Teo et al. [62] used virtual hands and a rectangular frame as a visual cue to enhance co-presence for better collaboration. 


\subsection{Chapter Summary}

In chapter 2, we reviewed the concept of presence and collaborative works in a virtual environment (VE). We also highlighted that the majority of related works have focused on the interaction between groups, not within the groups themselves. Previous approaches to a co-located multi-user system for collaboration have mainly focused on virtual reality (VR) rather than leveraging mixed reality (MR), and required every user to wear HMDs or shutter glasses to interact with the virtual world. In addition, there is limited work with user studies to measure the system with regard to the spatial presence or social presence during collaboration. In the following chapter, we address this gap and propose our platform, which overcomes the previous limitations of CVEs for multiple users by providing a gate portal system to take advantage of the characteristics of large-scale displays in MR. 


\section{Chapter 3}

\section{TeleGate}

To overcome the previous limitations on CVEs for multiple users, we focus on the collaborative aspects of co-located users and how they interact together when using large-scale displays. In this chapter, we propose TeleGate, a novel multi-user teleportation platform for remote collaboration in a MR space. We start this chapter by introducing the concept of "gate portal" that provides "semi-teleportation" into remote workspace. Then we explain how we implemented this concept using the large-scale displays through a system overview. Lastly, we demonstrate what challenges we faced with supporting the collaboration of multiple users and how we solved them regarding camera and object control.

\subsection{Concept Overview}

In this overview, we describe the concept of a "gate portal" that enables semi-teleportation (Figure 3.1). First, we define multiple users located in the same space for teleporting together as "teleporters". The gate portal separates a collaborative environment into two spaces: communication space and workspace. While the teleporters' bodies physically remain in their physical space where they are actually located (referred to as communication space), they "semi-teleport" together to another remote location 


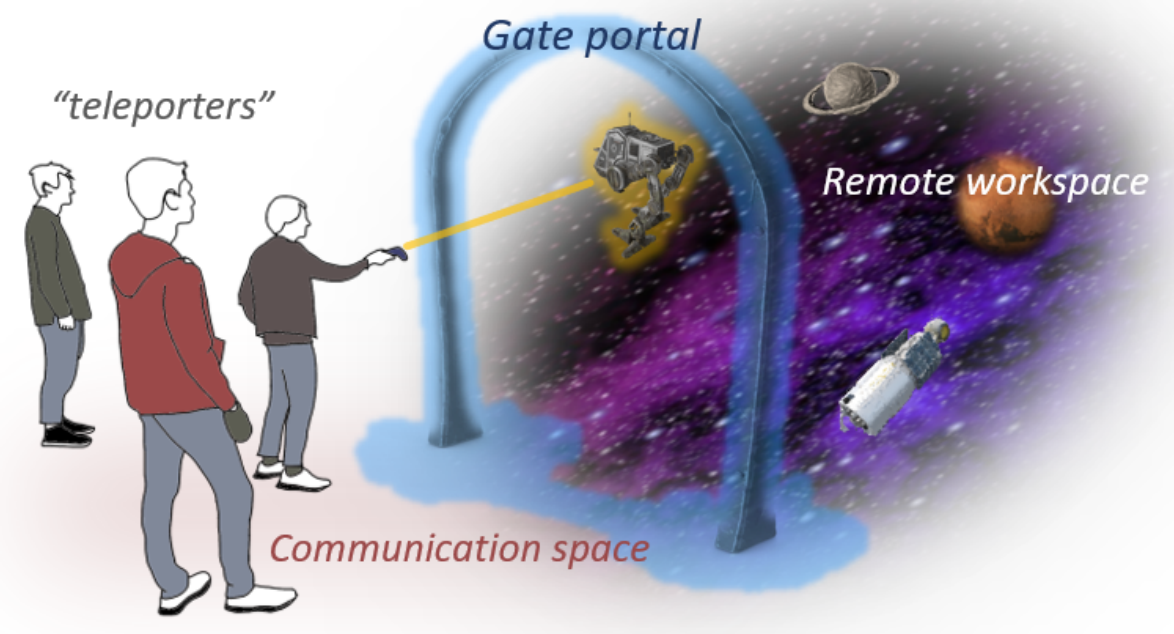

Figure 3.1: Overview of the gate portal concept.

for collaboration (referred to as remote workspace). Semi-teleportation implies that teleporters perceive both their physical communication space and the remote workspace at the same time. As teleporters do not enter the remote workspace but are still located in the local space, they can influence the workspace only through the gate portal. Thus, the use of pointers enables teleporters to interact with the remote workspace, helping them to reach the objects over the gate portal regardless of each teleporter's position in the local communication space. In summary, the gate portal allows teleporters to be aware of the remote workspace's surroundings and feel spatial presence in that space while maintaining natural communication.

For the purposes of this thesis, we define the physical communication space as a local space where multiple users are positioned side-by-side around the TeleGate to engage in collaboration. The remote workspace, on the other hand, is referred to here as a MR collaboration (MRC) space, which is the virtual environment where the results of users' actions are realised. In our setup, multiple users are not entirely surrounded by a 
VR environment, but share the view of the MRC space through TeleGate streamed by a 360-camera, while still being in the same local space with other users. Multiple users can participate in the collaboration at the same time in the MRC space together by interacting with objects, enabling realworld references for MR collaboration. They can perform collaborative tasks by manipulating augmented virtual objects seamlessly blended into the MRC space, enabling them to semi-teleport into the MR environment. By using TeleGate, natural interaction is preserved between multiple users, which enables them to conduct the tasks together while feeling a sense of spatial presence in the MRC space. 


\subsection{System Overview}

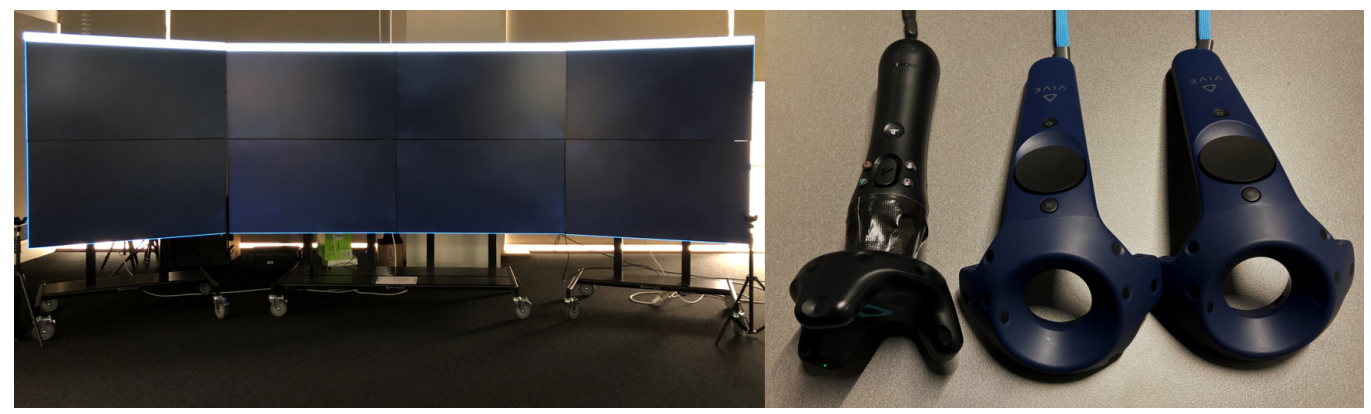

Figure 3.2: Left: Large-scale video-wall displays to act as a gate portal between the local space and the remote space.

Right: 6-DoF motion controllers to capture the users' movement for changing viewpoints and manipulating objects.

In this section, we describe a system overview of TeleGate for multiuser semi-teleportation for remote collaboration in MR 360-videos. We achieved the concept of TeleGate using large-scale video-wall displays to act as a gate portal between the local space and the remote workspace. These displays are formed in a cylindrical shape, creating improved spatial presence and user experience comparing to flat-based setups (Figure 3.2.Left).

We implemented the MR collaboration (MRC) space using 360-videos with seamless blended virtual objects to enable a minimal gap between the virtual world and the real world. By locating a 360-camera anywhere in the remote site, the remote surrounding environment is easily acquired from a viewpoint where the camera is located in real time, providing a high-fidelity scene.

We used the Unity 3D game engine to implement the platform using a client-server protocol. This setup allows for an encoded stream to be captured by a client running on the 360-camera that sends the data through the network using an Nginx server with an RTMP module. This is then re- 
ceived by the client program, which is able to display the 360-videos into the Unity 3D scene virtual environment using the Vive Media Decoder.

Regarding lighting and composition, we used the method described in the MR360 paper [52] in Unity engine to seamlessly compose virtual objects into the 360-videos. Multiple users can watch the rendered remote place with composed objects in the large-scale display that provide an immersive experience.

To interact with the MRC space and augmented objects, we provided six degrees of freedom (6-DoF) motion controllers to capture the users' movement for changing viewpoints and manipulating objects. We used Vive trackers placed underneath the displays to recognise the position of the displays in the real world. Once the position is recognised, multiple users can change the perspective of MRC space and interact with virtual objects by using Vive controllers or PS4 motion controller with an attached tracker (Figure 3.2-Right).

In the following section, we describe how we implemented the TeleGate platform (Figure 3.3) for collaboration in terms of camera and object control in more detail. 


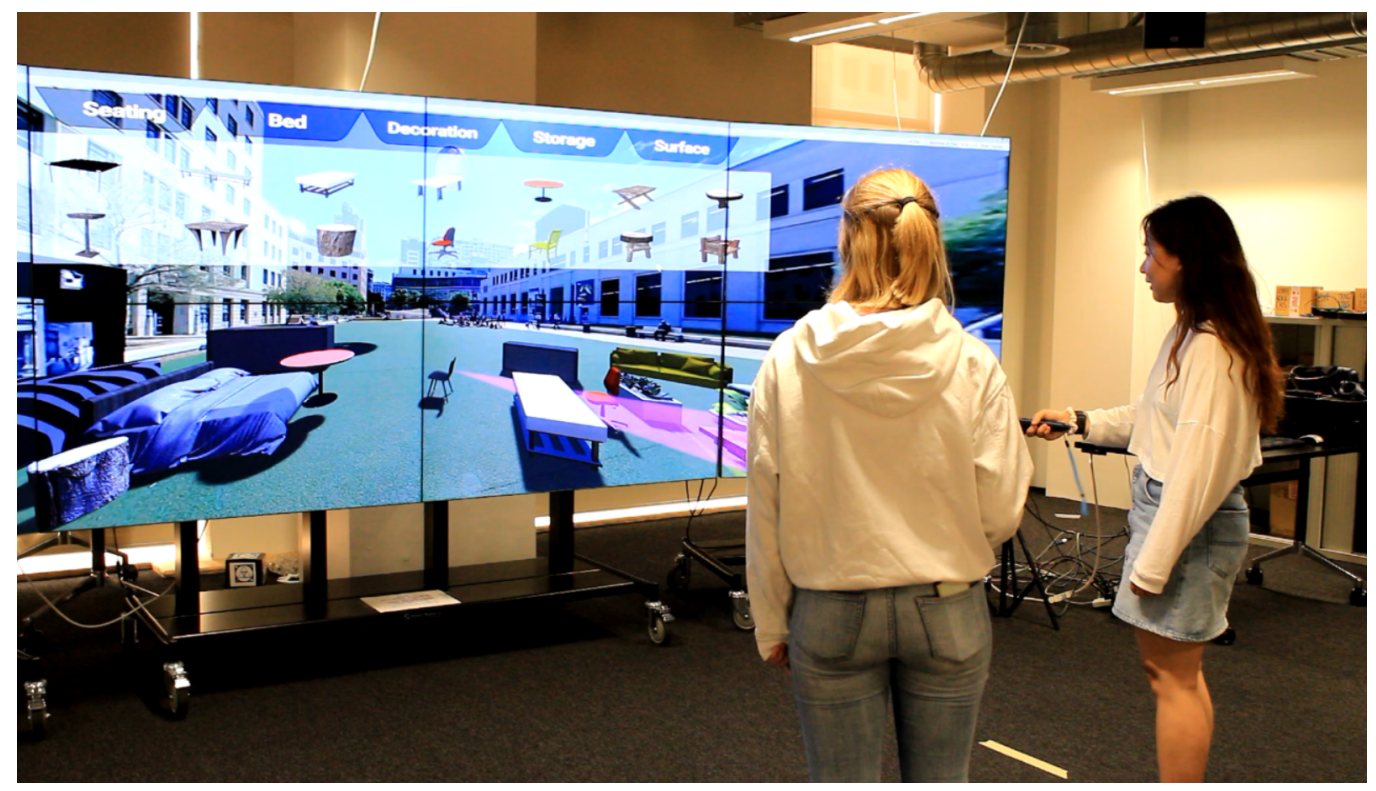

Figure 3.3: Overview of the TeleGate working prototype. 


\subsection{System Implementation}

We implemented the concept of TeleGate using the large-scale displays that connect the local and remote space, and then a remote MRC space was live-streamed as 360-videos with seamless blended virtual objects. Multiple users can act on these virtual objects by selecting or manipulating the objects, while still maintaining a reference for the real physical space in the MRC space. However, it is challenging for large-scale displays to support separated viewpoints individually; we provided physical controllers to individual users to change the viewpoints of the MRC space. Furthermore, since multiple users still stay in their local space but influence the workspace only through TeleGate, the given controllers support users to interact with augmented virtual objects for manipulation. Therefore, our implementation consists of three parts: controller design, camera control, object control.

\subsubsection{Controller Design}

To enhance face-to-face collaboration and interaction performance [10, 35], we provided a physical controller to each user as a wand. The controller supports users to change the viewpoints of the MRC space and interact with the virtual objects. In order to capture each user's movement, we used Vive device and streamed to the Open VR application. This is because compared to an optical motion tracking system, Vive controllers are cost-effective to track the users' movement.

As the Vive device supports two controllers; we utilised an additional controller that can substitute for standard Vive controllers for the situation when more than two users are collaborating. The additional controller was a combination of a Vive tracker and a PS4 motion controller that has a similar appearance as Vive controllers to map the button (Figure 3.2-Right). We tracked each user's movement from the Vive tracker with 6-DoF along three axes and obtained the user's input from the PS4 motion controller 
via Bluetooth. To mock up the feature of the standard Vive controllers, we took advantage of the multiple buttons on the controller to manipulate the virtual objects by only using a single physical controller.

The controller was designed for more than one user to directly interact with our platform at the same time. Users were able to manage the platform by simply pressing the button on the controller and moving it. Manipulating incorporates four buttons: grip button, trigger, pad button, menu button (Figure 3.4). A grip button positioned on the side allowed users to control the orientation of a camera when pressed down. A trigger button supported selecting the virtual object while being held down. A large circle pad button on the top of the controller enabled object rotating or scaling when it is pressed simultaneously with the trigger button and moved from side to side. A small menu button above the pad button toggled the mode between rotation and scaling.

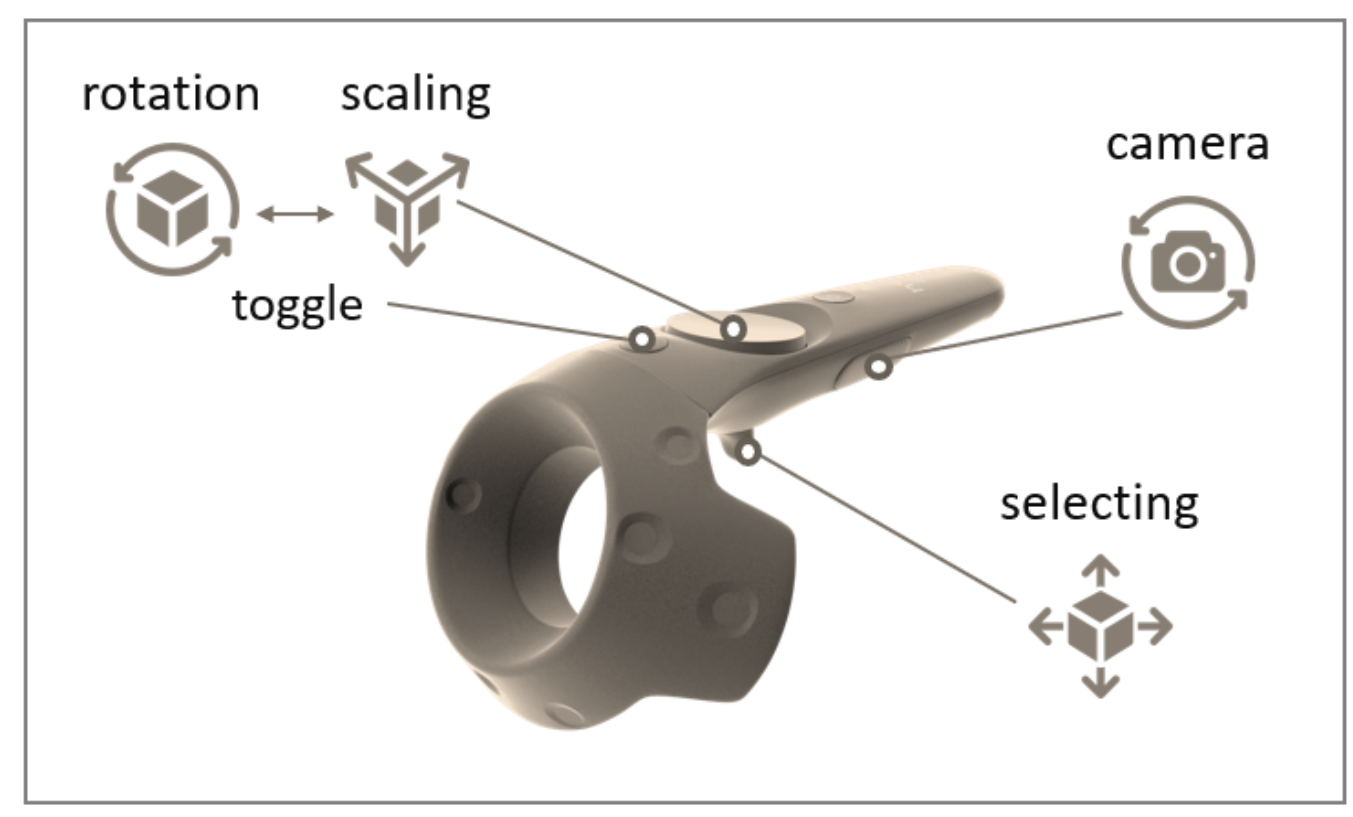

Figure 3.4: Manipulating incorporates four buttons: grip button, trigger, pad button, menu button. 


\subsubsection{Camera Control}

360-cameras offer omnidirectional background, recording the remote scene where the camera is located. Perceiving precisely the remote spatial information is ambitious without a $3 \mathrm{D}$ viewpoint of the scene [1]. For this reason, we gave users the freedom to explore the MR environment in the fixed position for better understanding of the remote MRC space. Any user who takes over the controller can change the viewpoints. By doing so, multiple users can have an omnidirectional viewpoints to understand the 3D spatial information simply by changing the perspective of the camera.

\section{Camera Setup}
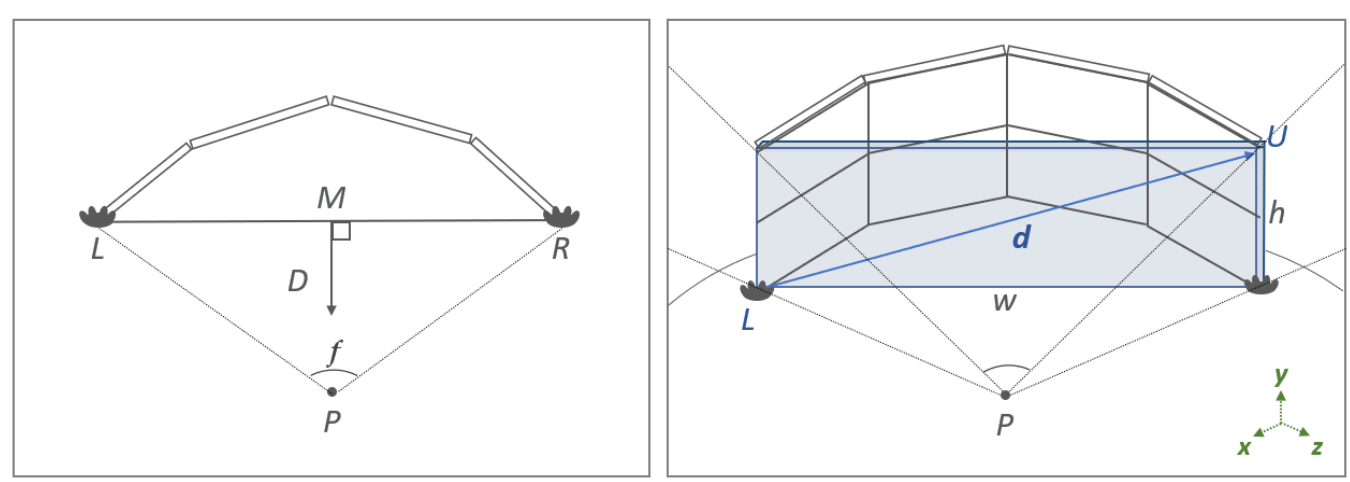

Figure 3.5: Two Vive trackers placed on each lower corner of the displays to track the displays in the real world for creating a virtual screen and positioning the virtual camera.

In order to show users 360-videos through the displays, a virtual camera was created to render the scene in the Unity engine. We recognised the position of the large-scale displays to locate the virtual camera in the appropriate position for the rendering.

To track the displays in the real world, we placed two Vive trackers on each lower corner of the displays using tripods, respectively [68]. We 
denoted the left corner tracker as $\mathrm{L}$ and right corner tracker as $\mathrm{R}$ (Figure 3.5). Then, we can get the position of the right upper corner (U) since we knew the height of the displays. The virtual screen representing the largescale displays was generated as a box collider as follows:

1. Calculate the middle point between $L$ (left lower corner) and $U$ (right upper corner).

$$
P=L+\frac{U-L}{2}
$$

2. Calculate the scale factor of the box collider.

$$
\begin{gathered}
d=|U-L| \\
w=\sqrt{d^{2}-h^{2}}
\end{gathered}
$$

3. Calculate $\theta$ to rotate the box collider to align with the trackers.

$$
\theta=\arctan ^{2}([-1,0,0] \times \overrightarrow{U L} \cdot[0,1,0], \overrightarrow{U L} \cdot[0,1,0])
$$

In order to locate the virtual camera, we calculated a proper position (P) of the virtual camera in front of the virtual screen where the camera's horizontal field of view (FoV) fit the screen size. To find the P, we calculated horizontal FoV (f) using vertical FoV (v) and the displays' size through the following steps:

1. Calculate $\mathrm{f}$ (horizontal FoV of the camera).

$$
f=\frac{2 \pi}{360} \times 2 \times \arctan \left(\tan \left(\frac{v \times \frac{2 \pi}{360}}{2}\right) \times \frac{w}{h}\right)
$$

2. Calculate $\theta$ to rotate the camera to align with the virtual screen.

$$
\theta=\arctan ^{2}([-1,0,0] \times \overrightarrow{R L} \cdot[0,1,0], \overrightarrow{R L} \cdot[0,1,0])
$$

3. Calculate $M$ (the middle position) between $\mathrm{L}$ (left centre side) and $\mathrm{R}$ (right centre side).

$$
M=L+\frac{R-L}{2}
$$


4. Calculate the distance between $\mathrm{P}$ (the position where the camera should be located) and $\mathrm{M}$.

$$
D=\frac{\left|\frac{R-L}{2}\right|}{\tan (f / 2)}
$$

5. Calculate the vector perpendicular to the screen that points towards P.

$$
\overrightarrow{P M}=\frac{R-L}{|R-L|} \times[0,-1,0]
$$

6. Find $\mathrm{P}$ (the position of the camera).

$$
P=M+(D \times \overrightarrow{P M})
$$

Consequently, by locating the camera at the position (P) according to the calculation above, the camera's FoV could cover the displays. Finally, the near clipping plane was adjusted to match the displays' size as the Unity engine supports adjusting the plane according to the distance (D) calculated.

\section{Camera Rotation}

All the 360-videos were rendered to a skybox in Unity engine showing how the 360-worlds look like beyond geometry. As the position of the skybox was fixed, the camera needed to rotate inside the skybox. We created one invisible game object to attach every component associated with the camera so that it can be rotated simultaneously when users rotate the camera.

At first, we implemented the virtual camera to be rotated when the direction was given by time. However, we figured out that changing the viewpoints by time was uncomfortable to use from the pilot test. Hence, we deployed the concept of "Scene in hand" technique [65] as if each one is holding the camera in his hand. The camera rotates along with the controller only as far as the controller is twisted by adding updated rotation 
directions to the pivot. When the user pressed the button, the previous orientation of the controller kept being updated to a new orientation value. When released, the camera angle remained fixed without having to return to the initial position. Thus, each user could change the perspective for themselves by pressing the trigger of the controller down and moving it.

Finally, we assumed that if the displays reflect the immoderate movement of the camera sensitively, it could cause simulator sickness for users while watching the MRC space. Therefore, we limited the camera control along the vertical and horizontal axis, in order to prevent side effects of cybersickness.

\subsubsection{Object Control}

Multiple users can interact with blended virtual objects in the MRC space using their controllers. When multiple users are given a specific goal, they can achieve the task by selecting or manipulating the virtual objects of interest. To support natural pointing and selection of the objects [57], we employed pointing ray. The pointing ray is visualised as a virtual line segment attached to the controller to define the direction of pointing [38]. This pointing ray also enables better performance in selecting objects regardless of the user's position in the virtual world [16, 38] and supports multiple users to see each one's interaction with the objects. In the last section, we illustrate how we implemented the natural pointing ray using a ray-casting method for manipulating objects.

\section{Ray-Casting}

In terms of object control using the large-scale displays for multiple users, special ray-casting was required as users are sharing single displays for interacting together. As shown in Figure 3.6, where the camera was located to render the scene and where each user stayed in the real world was different, which resulted in distortion in seeing the pointing ray. The 


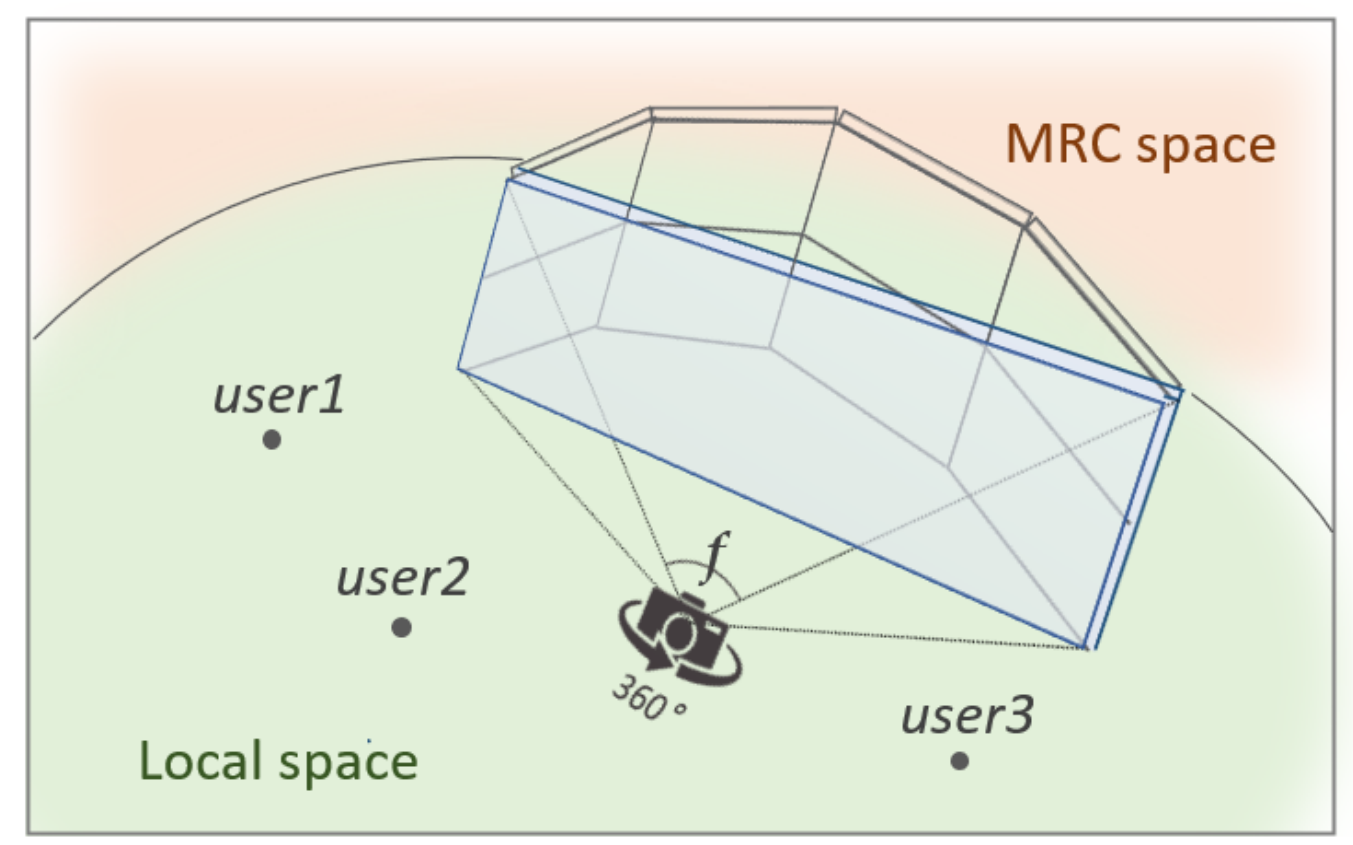

Figure 3.6: Discordance of the position between the camera and each user.

pointing ray needed to be rendered with the correct perspective according to each user's position as if it came from the user's position towards displays. Thus, we redirected the orientation of the pointing ray by using two auxiliary rays to match where the camera rendered the scene in the virtual world and where each user was located in the real world (Figure 3.7.

First, we detected whether the virtual ray hit the virtual screen or not. When the user pointed to a virtual object on the screen using one's controller, an auxiliary laser (r1) was emitted from the controller as a line. When the $\mathrm{r} 1$ hit the screen, the point where $\mathrm{r} 1$ intersected the screen was stored to create a second auxiliary ray ( $\mathrm{r} 2)$. The $\mathrm{r} 2 \mathrm{was}$ created from the virtual camera's position towards the intersected position. Even though any number of objects hit by a single ray can be cast, we adjusted the program to grab the object behind the screen to be selected since every object was placed behind the screen. 
Then, when the $\mathrm{r} 2$ hit the virtual object passing through the screen, we got another intersected position on the object's surface to create the final ray $(r)$ from the controller towards the intersection. Even though the r2 was unable to reach the virtual object, the $r$ was created from the controller heading to the edge point of $\mathrm{r} 2$ so that the ray could still remain on the screen indicating where the user pointed at. This ray only can be seen when the users successfully hit the displays correctly; otherwise, nothing would appear on the screen.

Consequently, the final ray (r) was rendered between the position of the controller and the object that it hit. By doing so, users can select virtual objects accurately and see the pointer ray as if it comes out from their position to the displays regardless of where they are. Furthermore, in order to differentiate the pointing ray between users, we rendered each of the user's ray in a different colour and positioned a sphere cursor in the object when the ray hit the object. This can help users to identify which elements belong to themselves or others in the shared displays environment.
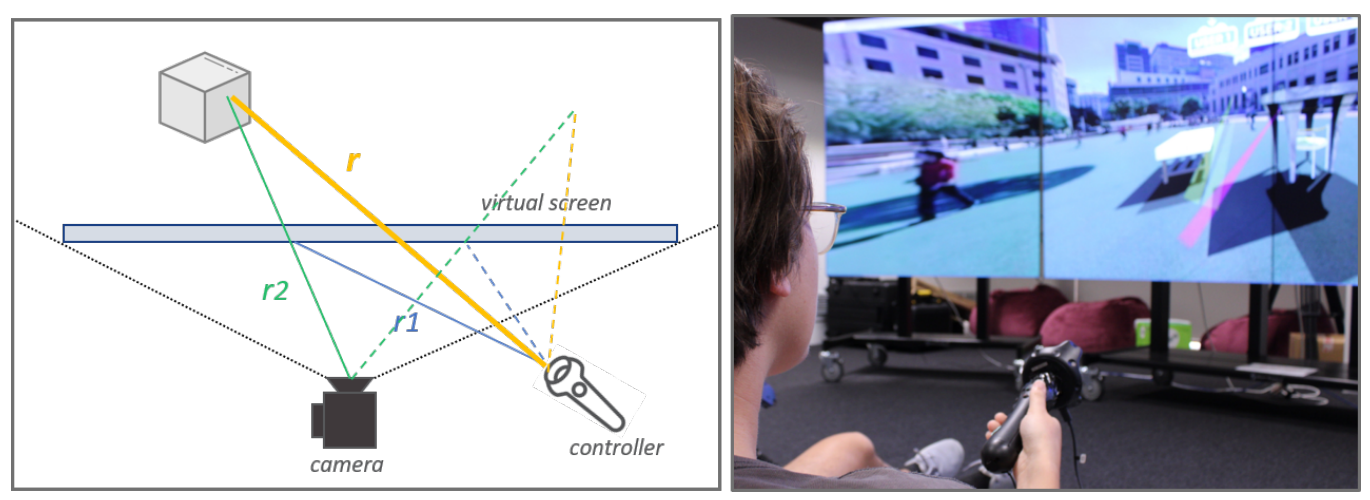

Figure 3.7: Explanation of the correction performed on a user's pointing ray. To enable the corrected ray's perspective from the user's viewpoint, first we perform a ray-casting between the controller's forward ray and the screen ( 1 1), then we project this point into the camera (r2), where we perform the ray-casting using the corrected perspective (r). 


\section{Object Manipulation}

Object manipulation is classified into three types: positioning, rotation, scaling.

First, the positioning technique is the task of moving an object from a starting location to a target location. The objects can be positioned depending on the movement of the users, which follows the pointer ray coming out from the controller. To locate the object far away or near the user's position, they need to push or pull the controller. The controller's previous $\left(P_{p}\right)$ position and new position $\left(P_{n}\right)$ were used to determine how far the object needed to be placed. The position of the object followed the distance (D) by calculating as follows:

1. Calculate a vector between the updated position $\left(P_{n}\right)$ and previous position $\left(P_{p}\right)$.

$$
\vec{V}=P_{n}-P_{p}
$$

2. Project the vector onto 2D space (x-z plane) and calculate the length.

$$
\Delta=|\vec{V}|
$$

3. Calculate the direction to check whether the controller moved forward or backward.

$$
\operatorname{Dir}=\frac{\vec{V}}{|V|} \cdot[0,0,1]
$$

4. Decide the direction by converting to -1 or 1 .

$$
\text { Dir }=\left\{\begin{aligned}
1, & \text { Dir }>0 . \\
-1, & \text { otherwise }
\end{aligned}\right.
$$

5. Calculate the distance.

$$
D=\Delta \times \operatorname{Dir} \times \varepsilon
$$


To make this positioning interaction easier, the snapping function was implemented where the object was automatically attached to the target position when it reached the collider of the target position. Also, we prevented objects from falling under the ground plane where the shadow of the objects rendered so that they can keep being tracked to preserve the shadow mapping.

Secondly, the rotation technique is the task of changing the orientation of an object. To make simple control of the interaction, we fixed the orientation except for $y$-axis, allowing the object to spin along only left and right. Lastly, the scaling technique is the task of changing the size of an object. We limited the size of the object to be scaled up and down between 0.5 and 2 based on the initial size. 


\subsection{Chapter Summary}

In chapter 3, we proposed TeleGate, a collaboration platform that enables multiple users to semi-teleport together. In the concept overview, we defined a physical communication space as the local space and remote workspace as the MRC space. In the system overview, we explained how TeleGate was achieved using the large-scale displays with the 360-camera for highfidelity with blended virtual objects. In order to support multiple users to interact with our platform, we provided physical controllers to individual users. Using controllers, users can change the viewpoint of the MRC space for getting spatial information and manipulating the virtual objects in 360videos with natural pointing and selecting. Conclusively, TeleGate allows multiple users to semi-teleport together in remote MRC space for collaboration in object manipulation while maintaining natural communication. We investigated this novel concept of teleportation with a user study. In the next chapter, we present the user study to analyse our proposed platform with collaborative tasks between groups of people in a co-located environment. 


\section{Chapter 4}

\section{User Study}

We conducted a user study to evaluate a working prototype of TeleGate that allows multiple users to semi-teleport together in the remote MR collaboration (MRC) space. In contrast to existing works that revolve around a single user interacting with a remote user, we focused on how individual users within a co-located group interact with one another and feel a sense of presence in the remote space together. We begin this chapter with a section stating our research questions, and then describe the evaluation design, the methodology, and the result of the user test.

\subsection{Research Question}

We investigated TeleGate that enables "semi-teleportation," which is the ability of directly interacting in the MRC space while being able to collaborate effectively in the same physical local space. To evaluate this concept, we measured the sense of spatial presence and social presence in both spaces - MRC space as well as local space - while collaborating. Additionally, the current setup enables more than one user to share and interact with the system simultaneously. Thus, it is essential to investigate how different control mechanisms can be used to facilitate collaboration. Therefore, we defined the following research questions: 
RQ1) Between the MRC space (where they semi-teleport) and the local space (where they are physically located), in which space do multiple users feel a presence?

RQ2) What kind of impact do different control mechanisms have on improving co-located collaboration for multiple users?

Since users' actions are performed in the MRC space, we hypothesised that the spatial presence would be higher in the MRC space when compared to the local space where they are located. Also, we argue that allowing users to collaborate in their physical space as communication space would enable high social presence in both spaces when they can actively perform actions in the MRC space. The degree of control over the circumstance is positively related to presence, as the more control users have in interacting with a virtual environment, the better their experience of presence [24, 70]. Thus, enabling each user to have control would benefit co-located collaboration, compared to where one user controls the space alone. Based on these assumptions, our research hypotheses are summarised as follows:

H0: Multiple users would feel a better spatial presence in the MRC space where they work together when compared to their local space.

$\mathrm{H} 1$ : Social presence would be high both in the MRC space and the local space, when users have some control over the environment.

$\mathrm{H} 2$ : The scenario where all users have some control over the environment would benefit co-located collaboration.

The following section addresses how the evaluation is designed to answer these hypotheses. 


\subsection{Evaluation Design}

\subsubsection{Experimental Design}

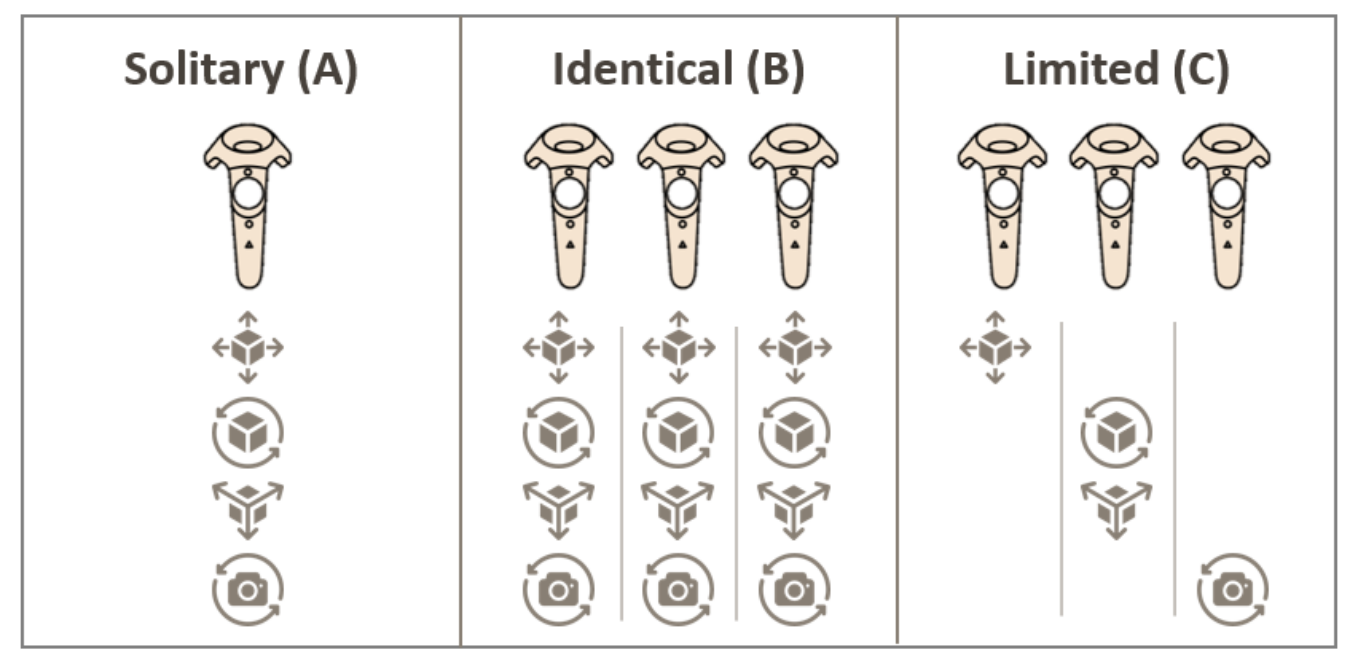

Figure 4.1: Three different control mechanisms between each group of users enabling different collaboration strategies.

To answer the above research questions, we varied the level of control between users in a classic object manipulation task, where users are able to control camera movements, position, rotation, and scale of virtual objects. We then designed three different control mechanisms between each group of users enabling different collaboration strategies (Figure 4.1): Solitary control (A), Identical control (B), Limited control (C).

Solitary control enables a single user to control the system; on the other hand, identical and limited control allows every user to participate in an object manipulation task directly. In solitary control, a single user has full control of the interaction, while the other users are only able to collaborate using voice and body-language to advise what to do. However, identical control gives all users the same degree of full control, which may cause conflicts in the collaboration. To prevent users from interfering with each 


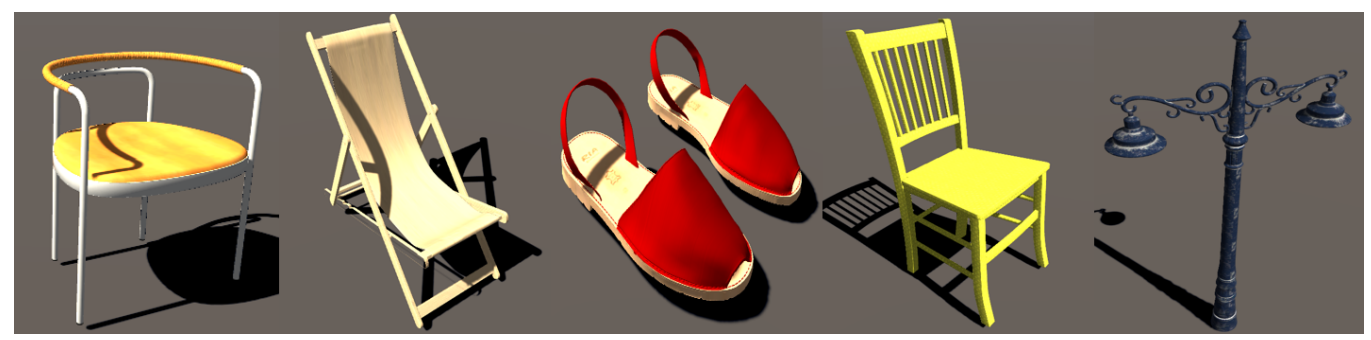

Figure 4.2: Example of the reference paper given to each group of participants which indicates five target objects.

other's actions caused by having the same controller, limited control enabled every user to have a controller, but each of them performed one degree of operation at a time. Each of the users had a specific role, with one of them being able to perform object selection and positioning, another one rotation/scaling, and a third camera control.

The experiment was conducted in a controlled environment, where we evaluated both presence and control mechanisms using a within-subjects design with a balanced Latin-squared design to avoid bias.

\subsubsection{Task Design}

We designed a collaborative task that combined a search task with a 3D object manipulation task for participants to collaborate in the MRC space. The goal of the task was to search for the target object to place it at the given spot, and to adjust it to the given scale and rotation.

Before conducting the task, we provided a reference paper to participants with a picture of target objects that needed to be found (Figure 4.2). Participants were asked to find 5 target objects among scattered distractors in the MRC space and move them to indicated points. Then, virtual indicators supported participants in understanding the transform information for adjusting the scale and rotation of the target objects.

To mitigate learning effects, we presented three kinds of reference papers made up of different pictures in a different order to each group and 
the position, rotation, and scale of the target object were randomised in each trial. Moreover, each position of the virtual indicator was randomly set within 15 (metres) from the virtual camera, and the range of rotation and scale were defined as $0-360$ and $0.5-2.5$, respectively. In the following subsection, we explain how the virtual indicators were designed for the collaborative task.

\subsubsection{User Interface}

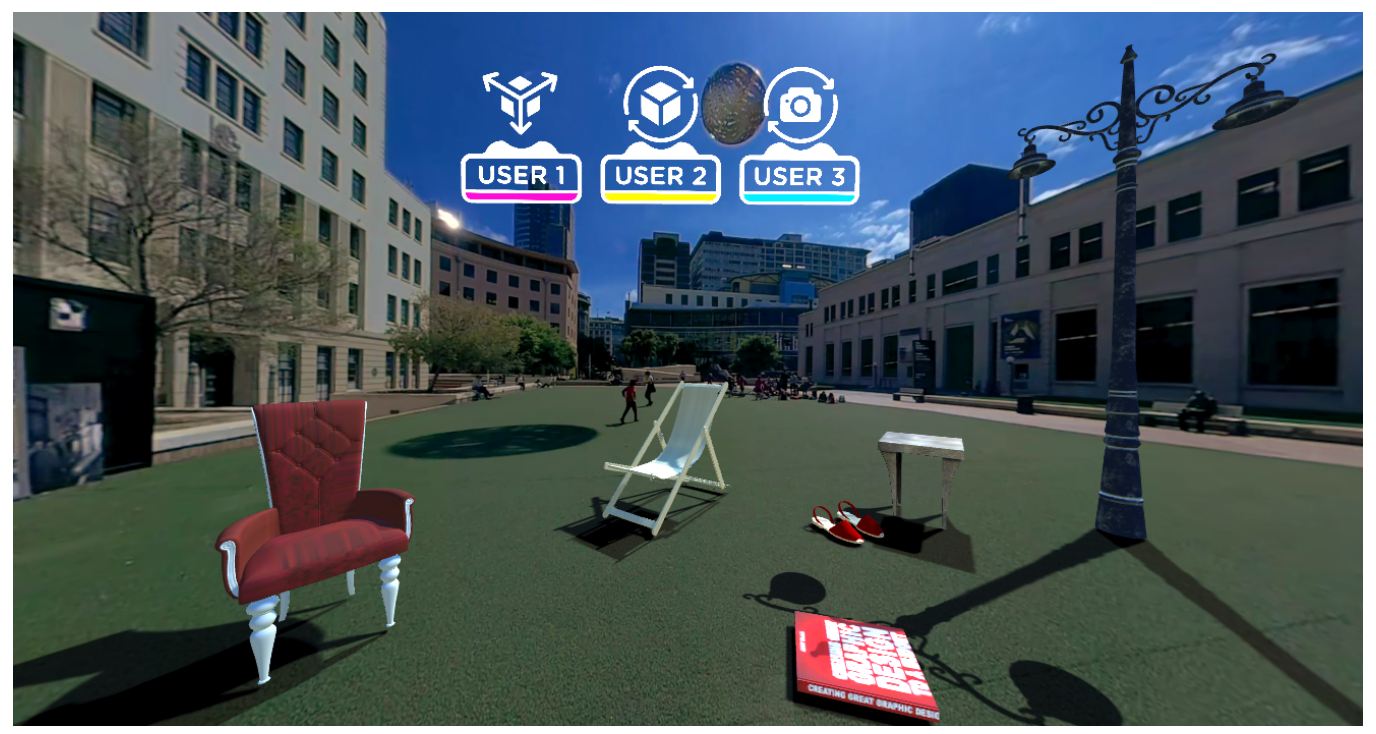

Figure 4.3: 2D user interface at the top of the large-scale displays to show each participant's state of the controller.

To assist the collaborative task, we designed two kinds of user interfaces to (1) facilitate controller manipulation and (2) provide reference information.

First, the 2D user interface supporting controller manipulation aimed to help users easily understand their and the collaborators' activity. We positioned 2D user interface at the top of the large-scale displays to show each user's state of the controller. The interface consisted of three pictures 
indicating whether the controller was in rotating mode, scaling mode, or camera mode (Figure 4.3). Each interface was assigned a different colour, helping multiple users to straightforwardly recognise their collaborators' role and their state. The position of the user interface is fixed, remaining in the same spot even though the camera is rotating.

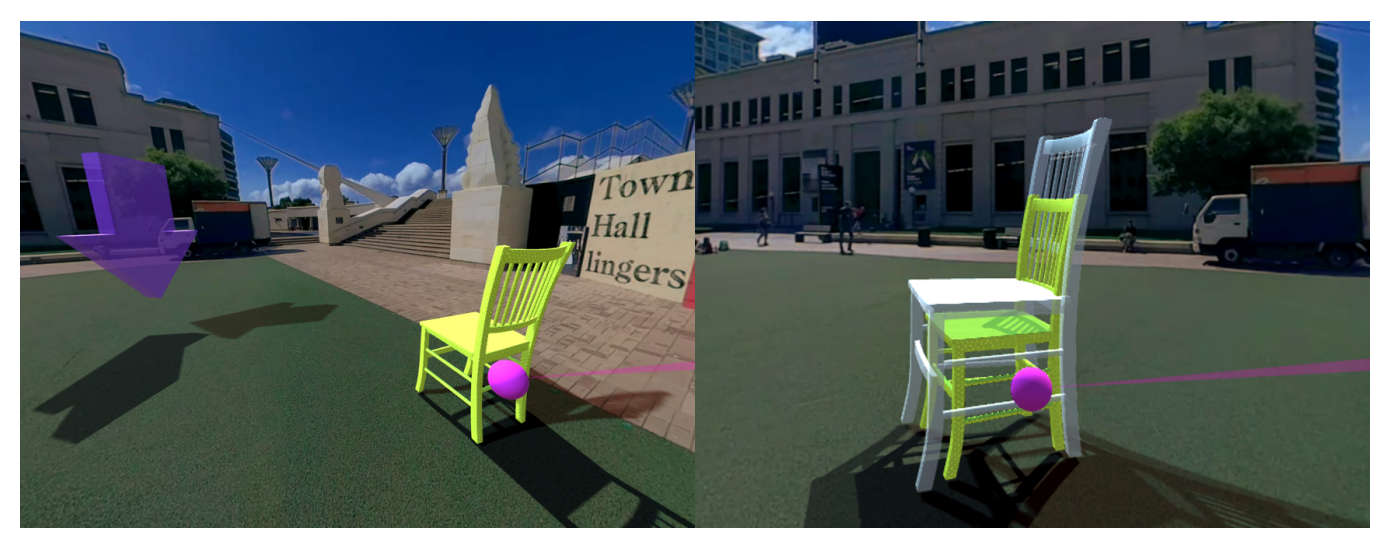

Figure 4.4: 3D virtual indicators as a reference to support participants.

Left: A 3D arrow pointing to where a target object should be relocated.

Right: A ghost object indicating correct rotation and scale.

Then, we provided 3D virtual indicators as a reference to support participants in accomplishing their task. These indicators appear when participants grab a virtual object and disappear when they release it. Firstly, as arrow type help enables users to effectively conduct a searching task in 360-videos [19], we used a virtual 3D arrow pointing to where a target object should be relocated. Every time participants selected the target object the virtual 3D arrow was rendered to indicate the given location. Secondly, we leveraged a grey translucent object as a ghost object to inform users what to do. Once participants placed the target object in the right spot, a transparent ghost object appeared to give information on the required degrees of rotation and the required size of scale (Figure 4.4). Therefore, participants could adjust the rotation and scale of the target object corresponding to the ghost object. Consequently, they were able to conduct the 
task intuitively without pre-education or memorising the goal of the task.

\subsubsection{Setup and Apparatus}

For the purposes of our user study, we used large scale video-wall displays which were composed of eight LCD displays (LCDs). These displays had a resolution of $1920 \times 1080$ pixels and were arranged in a $4 \times 2$ grid forming an arch, surrounding the users and thus creating a more immersive experience. To ensure maximum performance, we used a computer with Intel Xeon Gold 5122 CPU (Hz), Nvidia Quadro P5000 GPU, and 32 GB of RAM. To minimise latency caused by networking, we used a pre-recorded 360-video that was filmed outdoors using a Samsung 360 Round camera, instead of using live streaming.

Three bean bags were provided for the participants' convenience in the communication space. Participants could sit or stand side-by-side in front of the video-wall to communicate and interact with the MR environment.

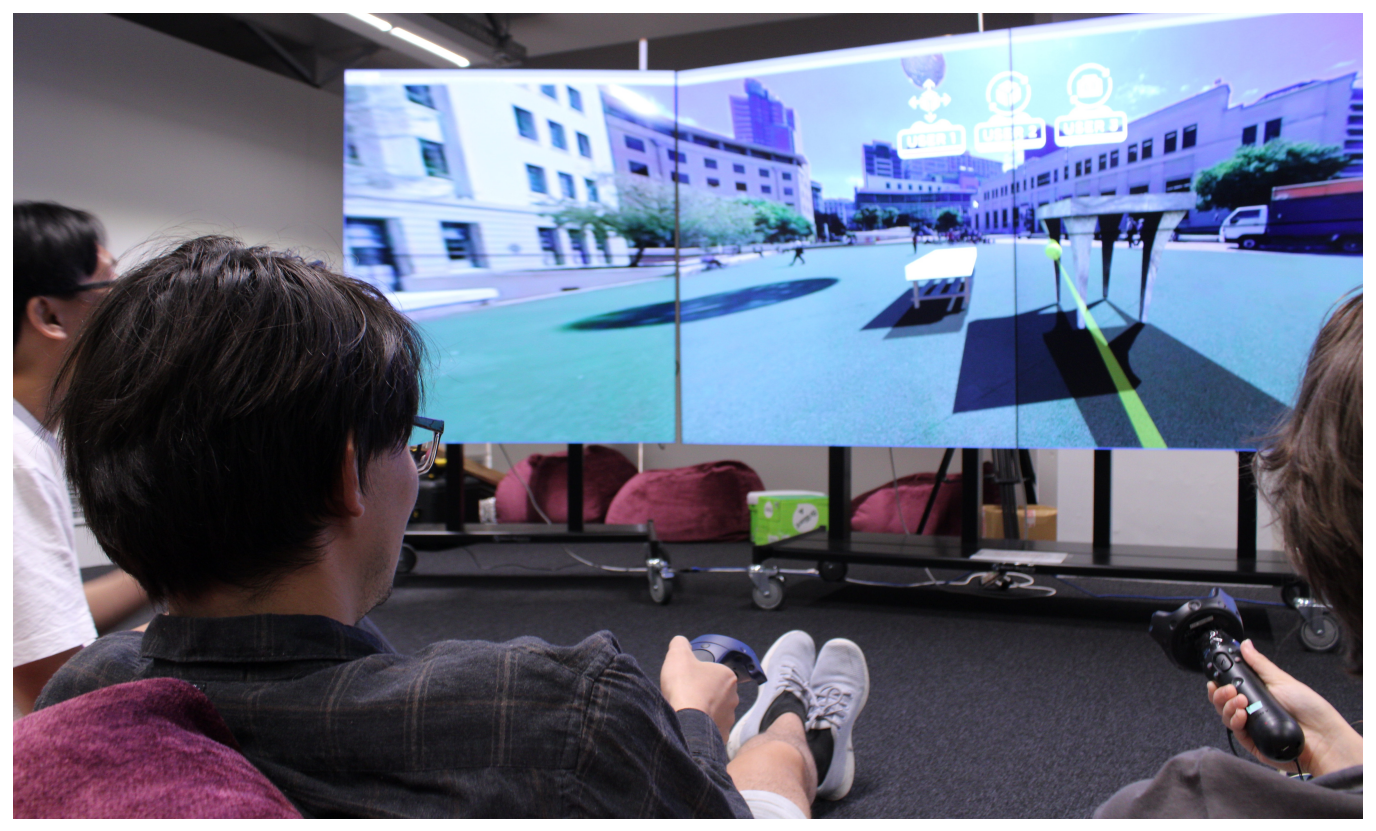

Figure 4.5: Three participants conducting the collaborative task together. 


\subsection{Materials}

To evaluate the presence in both the MRC space and the local space and user preferences of different control mechanisms, we used the following metrics:

\subsubsection{Spatial Presence}

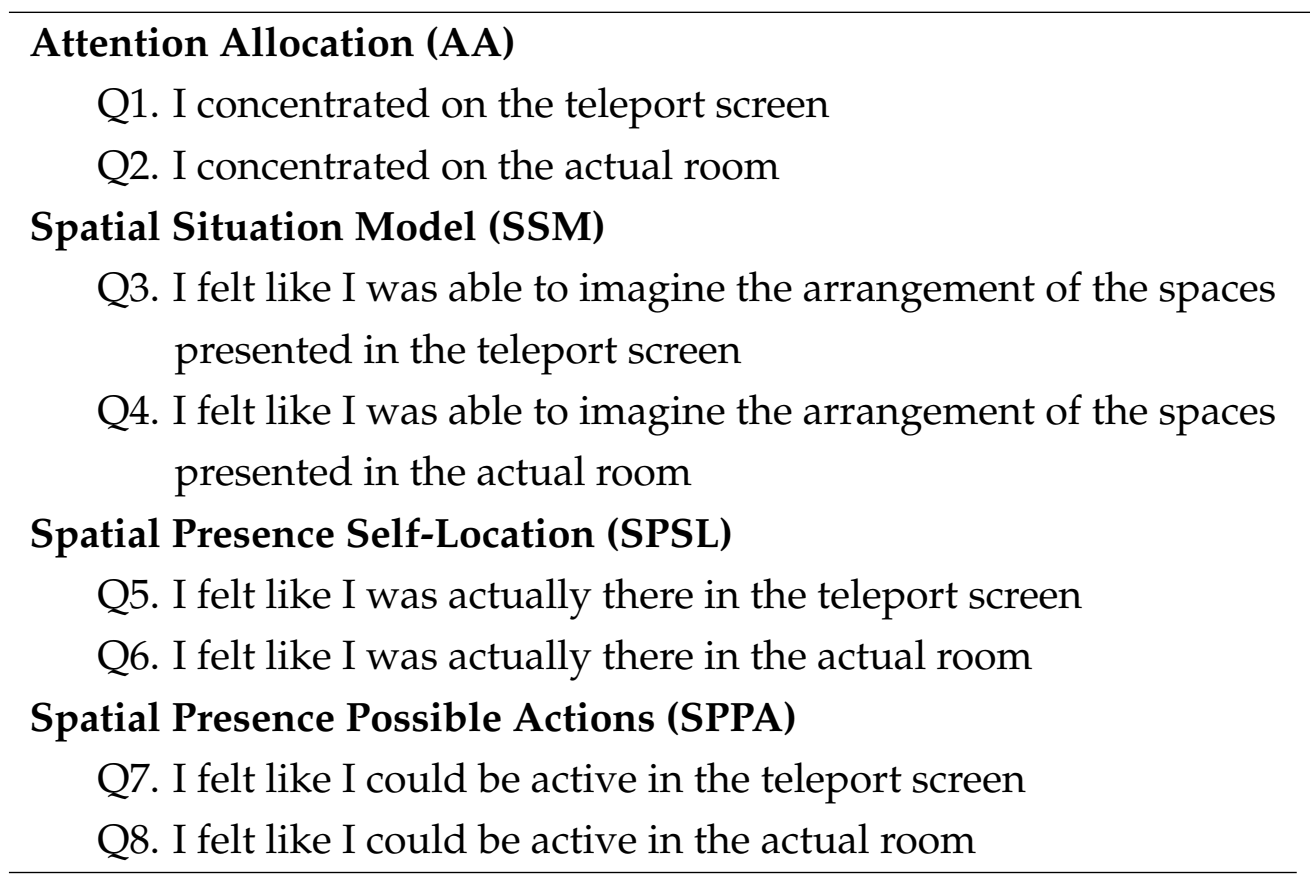

Table 4.1: Spatial Presence Questionnaire

To measure spatial presence in our setup, we adapted the questions from the MEC Spatial Presence Questionnaire (MEC-SPQ) [63] as shown in Table 4.1. We focused on two sub-scales of process factor of spatial presence: Attention Allocation (AA), which is the amount of attention one user gives to the other users and Spatial Situation Model (SSM), which measures how people process spatial cues and understand them in relation to space. In addition to this, we measured spatial presence formed by Spatial Pres- 
ence Self-Location (SPSL), which is the impression of being located in the virtual environment, and Spatial Presence Possible Actions (SPPA), which enable one to feel that they are acting in a mediated environment.

As a key aim was to investigate in which space users feel a better spatial presence, whether in the MRC space or in the local space, we divided the mediated environment into two spaces: teleport screen and actual room. The teleport screen represented the MRC space where the collaborative work happened, and the local room referred to the space where the users were physically located and communicated with each other.

\subsubsection{Social Presence}

\section{Impression of Interaction Possibilities (IIP)}

Q1. I was able to interact with other participants in the teleport screen

Q2. I was able to interact with other participants in the actual room

\section{Co-presence}

Q3. I was aware that other participants were with me in the teleport screen

Q4. I was aware that other participants were with me in the actual room

Table 4.2: Social Presence Questionnaire

To measure the social presence, we used the Social Presence questionnaire proposed by Poeschl-Guenther et al. [49] as shown in Table 4.2. As the collaborative task is designed to enable all users to interact with each other, we chose two sub-scales of social presence: one measuring the social presence regarding their interaction, referred to as Impression of Interaction Possibilities (IIP), which referred to how people perceive their being able to 
interact with other participants; and Co-presence, which is the feeling of being together with other participants in the same environment. In the same manner as with the spatial presence metric, we evaluated social presence in both spaces separately to figure out which space promotes users to have a better mediated conversation.

\subsubsection{User preferences}

Q1. It was easy to locate the objects

Q2. It was easy to rotate/scale the objects

Q3. It was easy to move the camera

Q4. It was easy to complete the task (Task easiness)

Q5. It was easy to coordinate movements with other participants (Coordination easiness)

Q6. Other participants were influenced by my performance (My performance)

Q7. Other participants' behaviour influenced my performance (Others' performance)

Table 4.3: User Preferences Questionnaire

To measure user preferences of the 3D interactions using our platform, we collected subjective user comfort rating data using a questionnaire regarding effectiveness, efficiency, and satisfaction (Table 4.3). Five questions were organised that asked how easy it was to manipulate the objects using the given controller and to complete the task as well as how challenging it was to coordinate opinion with other participants. Two questions were also asked related to performance, on how each of the participants rated their and others' achievement during the task. 


\subsubsection{Task Performance}

We used task performance metrics to measure the quality of particular tasks. We assumed that task performance was determined by two types of data: completion time and precision.

In the case of completion time, we compared which condition enabled participants to complete the task earlier. Completion time data was recorded, including the amount of time spent: (1) completing the entire task, (2) searching the target object, (3) locating the target object, (4) rotating/scaling the target object. To measure how precisely participants executed the task, precision data was collected as to the accuracy of the rotation and scale.

\subsection{Procedure}

Our user study was granted approval by the university ethics board. Participants were provided with a description of the study upon recruitment, and once they agreed to participate, a specific time was set for them to do the study. Prior to commencing the study, participants were again provided with an overall explanation of the study and explained the conditions and tasks to be performed and were asked to sign an informed consent. Then, we asked participants to respond to a pre-test questionnaire where they filled a profile questionnaire related to demographic information, previous experiences with VR/AR technologies, and a general tendency towards media such as movies and video games.

The session was composed of one training session and three test sessions. During the training session, the participants learnt how to use the controller, through a brief tutorial on manipulating some virtual objects. In the test sessions, participants performed collaborative work together and answered a post-task questionnaire about their subjective opinion after each trial. During the test sessions, we observed the behaviour of participants to identify how they communicate and collaborate using different 
control mechanisms and recorded observation log about participants' reaction to each collaborative scenario. When the entire session finished, we conducted a semi-structured interview, where participants provided their opinions about the system and their experience of interacting with it.

\subsection{Data collection and Analysis}

Both subjective data and objective data were collected for analysis.

Subjective data was collected in the form of 7-point Likert scale questionnaires for spatial presence, social presence, and user preferences. For this type of data, we conducted Friedman parametric tests with Wilcoxon Signed Ranks Test $(a=0.05)$ as post-hoc tests to find statistically significant results between each pair of samples. The Bonferroni correction (sig. = sig. /3) was applied to prevent data from resulting in incorrect statistical significance caused by multiple comparisons. We also used the neutral line, which was 4 of a 7-point scale, to determine if samples can be considered as having high scores in subjective ratings.

Then, objective data from the system log was collected to analyse the task performance such as task, search, manipulation time, and precision. We performed a Shapiro-Wilk test $(a=0.05)$ to check that the data were normally distributed, then applied Friedman parametric tests with Wilcoxon Signed Ranks Test $(a=0.05)$ as post-hoc tests. 


\subsection{Result and Analysis}

\subsubsection{Demographics}

We recruited 24 participants for our user study, split into eight groups of three participants each. From these groups, the majority knew each other and consisted mostly of staff and students from Victoria University of Wellington that had previous experience with computer graphics research (21 participants, or $87.5 \%$ ). The majority of participants were male (18 participants or $75 \%$ ), with age varying from 20 to $48(\mathrm{M}=25.7, \mathrm{SD}=$ 6.415).

When we asked a question about a relationship between participants in the same group using a 7-point Likert scale (Q. How well do you know other participants?), they stated they knew each other well $(\mathrm{Md}=6, \mathrm{IQR}=$ 2.75). Most of the participants reported having some previous experience with VR: 13 (54\%) of them were familiar with 3D virtual environments, while the other 11 (46\%) participants were not very experienced.

As we aimed to measure presence, we assessed participants' immersive tendency to find out how they easily tend to become immersive, using the Immersive Tendency Questionnaire (ITQ) [70]. Immersive tendency is of three types: games, focus, and involvement, and higher ITQ scores reflect a higher feeling of presence. However, we noticed that the immersive tendency of the participants was average $(\mathrm{Md}=4.5, \mathrm{IQR}=1.54)$.

\subsubsection{Subjective Responses}

We collected subjective responses about participants' overall experience regarding the spatial presence, social presence, and overall preferences through questionnaires. The questionnaires used contained 7-point Likert scale statements, where 1 meant that they did not agree with the statement and 7 that they agreed fully with it. In the following subsections, we detail the results obtained regarding the aforementioned metrics for spa- 
tial and social presence, and overall user preference for different control mechanisms (Table 4.4).

\begin{tabular}{|c|c|c|c|c|c|c|}
\hline \multirow{3}{*}{ Presence } & \multirow{2}{*}{\multicolumn{3}{|c|}{$\begin{array}{c}\text { Local space } \\
\text { Control mechanisms }\end{array}$}} & \multirow{2}{*}{\multicolumn{3}{|c|}{$\begin{array}{c}\text { MRC space } \\
\text { Control mechanisms }\end{array}$}} \\
\hline & & & & & & \\
\hline & Solitary (A) & Identical (B) & Limited (C) & Solitary (A) & Identical (B) & Limited (C) \\
\hline \multirow{5}{*}{$\begin{array}{l}\text { Spatial presence } \\
\text { AA } \\
\text { SSM } \\
\text { SPSL } \\
\text { SPPA }\end{array}$} & $4.0(1.5)$ & $4.1(1.4)$ & $4.0(1.0)$ & $4.3(4.0)$ & $4.8(1.4)$ & $4.8(1.5)$ \\
\hline & $3.0(2.0)$ & $3.0(2.0)$ & $3.0(1.0)$ & $6.0(1.0)$ & $6.0(1.0)$ & $6.0(2.0)$ \\
\hline & $4.0(2.0)$ & $4.0(2.0)$ & $4.0(1.0)$ & $5.0(2.0)$ & $5.0(2.0)$ & $5.0(1.0)$ \\
\hline & $5.0(3.0)$ & $5.0(2.0)$ & $4.0(3.0)$ & $3.5(2.0)$ & $4.0(2.0)$ & $4.0(2.0)$ \\
\hline & $4.0(3.0)$ & $4.0(3.0)$ & $4.5(1.0)$ & $3.0(2.0)$ & $4.0(1.0)$ & $5.0(2.0)$ \\
\hline \multirow{3}{*}{$\begin{array}{l}\text { Social presence } \\
\text { IIP } \\
\text { Co-presence } \\
\end{array}$} & $6.0(1.3)$ & $5.7(1.8)$ & $5.7(1.0)$ & $3.2(2.5)$ & $5.7(1.8)$ & $5.5(1.5)$ \\
\hline & $6.0(1.0)$ & $5.0(2.0)$ & $5.0(1.0)$ & $3.5(4.0)$ & $6.0(2.0)$ & $5.5(1.7)$ \\
\hline & $6.0(2.0)$ & $6.0(2.0)$ & $6.0(2.0)$ & $3.0(2.0)$ & $6.0(1.7)$ & $6.0(2.0)$ \\
\hline \multirow[t]{2}{*}{ User preferences } & \multicolumn{6}{|c|}{ Control mechanisms } \\
\hline & \multicolumn{2}{|c|}{ Solitary (A) } & \multicolumn{2}{|c|}{ Identical (B) } & \multicolumn{2}{|c|}{ Limited (C) } \\
\hline Task easiness & \multicolumn{2}{|c|}{$4.5(3.7)$} & \multicolumn{2}{|c|}{$5.0(2.0)$} & \multicolumn{2}{|c|}{$6.0(3.0)$} \\
\hline Coordination easiness & \multicolumn{2}{|c|}{$4.5(2.7)$} & \multicolumn{2}{|c|}{$5.5(1.0)$} & \multicolumn{2}{|c|}{$6.0(1.7)$} \\
\hline My performance & \multicolumn{2}{|c|}{$4.0(2.7)$} & \multicolumn{2}{|c|}{$5.0(1.7)$} & \multicolumn{2}{|c|}{$6.0(1.0)$} \\
\hline Others' performance & \multicolumn{2}{|c|}{$4.5(2.7)$} & \multicolumn{2}{|c|}{$6.0(1.0)$} & \multicolumn{2}{|c|}{$6.0(1.0)$} \\
\hline No. of participants & \multicolumn{2}{|c|}{24} & \multicolumn{2}{|c|}{24} & \multicolumn{2}{|c|}{24} \\
\hline
\end{tabular}

Table 4.4: Overall subjective rating results on 7-point Likert scale presented as Median (Interquartile range). AA (Attention Allocation), SSM (Spatial Situation Model), SPSL (Spatial Presence Self-Location), SPPA (Spatial Presence Possible Actions), IIP (Impression of Interaction Possibilities).

\section{Spatial presence}

For evaluating spatial presence, we selected four sub-scales of the spatial presence questionnaire [69]: attention allocation (AA), spatial situation model (SSM), self-location (SPSL), and possible action (SPPA). We used an additional metric to check the overall spatial presence that we define as an aggregated spatial presence on the scope of this thesis, which was the average of the four sub-scales selected for this study. We compared social 


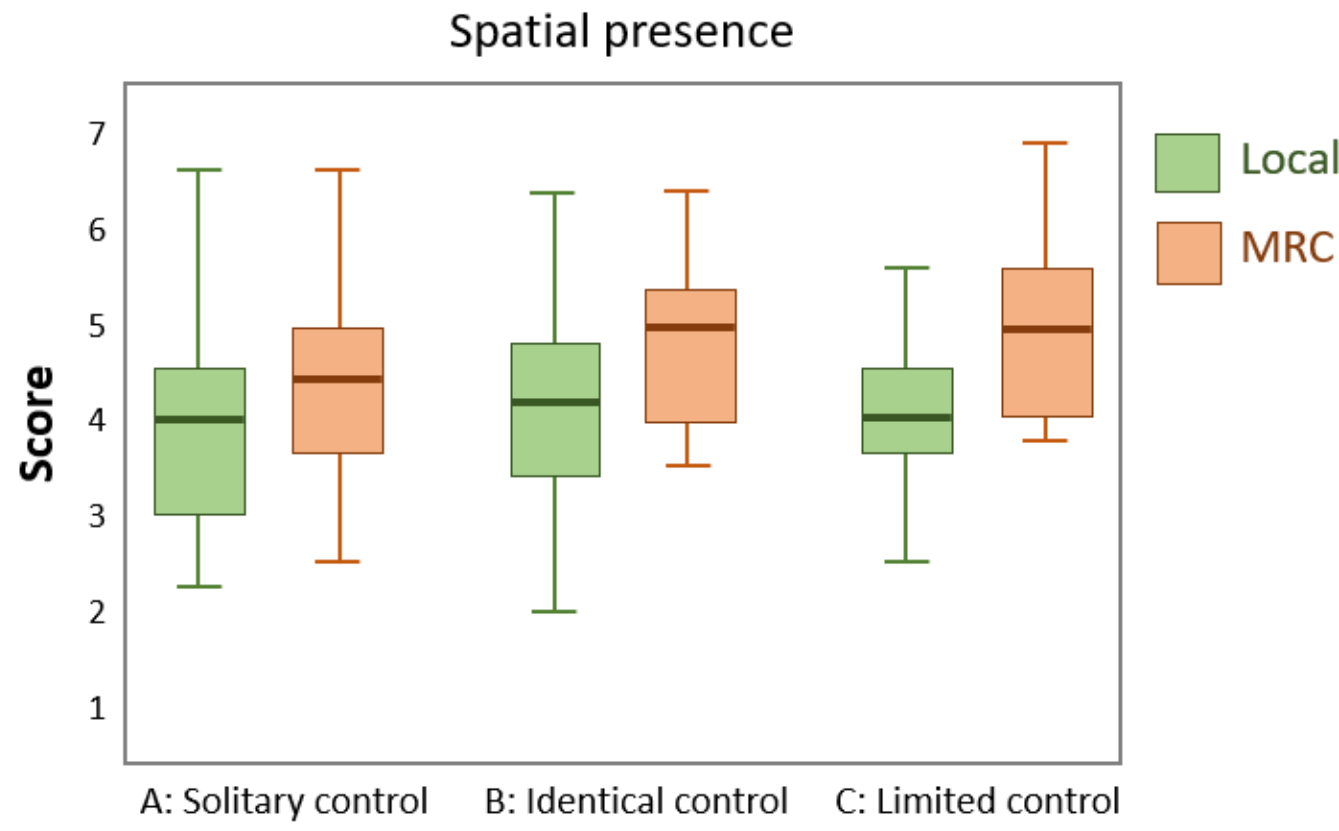

Figure 4.6: Spatial presence results between different control mechanisms. Green represents the local space, and orange represents the MRC space.

presence between the MRC space and local space using different control mechanisms.

We found statistically significant results in aggregated spatial presence between the two spaces (Figure 4.6). In this context, spatial presence in the MRC space was significantly higher than in the local space when all participants were allowed control over the platform. In identical control (B) condition, spatial presence in the MRC space was significantly higher when compared to the local space $(Z=-2.400, p=0.016)$. The same result was noticed in the limited control $(C)$ condition $(Z=-3.224, p=$ 0.001). However, no statistical difference was found in the solitary control (A) condition when only a single participant could control. We highlight that there was significant difference compared to the neutral line (B: $\left.\chi^{2}(2)=7.349, p=0.025, \mathrm{C}: \chi^{2}(2)=14.506, p=0.001\right)$, which indicated high spatial presence scores in the MRC space. This was particularly no- 
ticeable in both identical (B: $Z=-3.345, p=0.001$ ) and limited control (C: $Z=-3.540, p<0.0001)$. But no statistical significance was found for the solitary control condition compared to the neutral line.

We also analysed each sub-scale on spatial presence separately. Regarding attention allocation (AA), every control condition helped the participants to allocate their attention better in the MRC space than the local space (A: $Z=-4.026, p<0.0001, \mathrm{~B}: Z=-3.861, p<0.0001, \mathrm{C}$ : $Z=-4.182, p<0.0001)$, indicating significantly higher scores than the neutral line (A: $Z=-3.848, p<0.0001, \mathrm{~B}: Z=-4.204, p<0.0001, \mathrm{C}$ : $Z=-4.256, p<0.0001)$. In terms of spatial situation model (SSM), it revealed a similar result to the AA metric, which indicated statistically significant higher scores in the MRC space compared to the local space (A: $Z=-2.616, p=0.009, \mathrm{~B}: Z=-2.622, p=0.009, \mathrm{C}: Z=-3.436, p=0.001)$, resulting high scores as well (A: $Z=-2.863, p=0.004, \mathrm{~B}: Z=-3.270, p=$ $0.001, \mathrm{C}: Z=-3.670, p<0.0001)$. However, we found no significant difference in self-location (SPSL) and possible action (SPPA) between the two spaces except in the solitary control condition. While there was no significant difference between spaces in other conditions in favour of SPPA, solitary control resulted in statistically lower scores in the MRC space when compared to the local space $(Z=-2.400, p=0.016)$.

\section{Social presence}

We chose two sub-scales for evaluating social presence: impression of interaction possibilities (IIP) and co-presence [49]. Additionally, we used the aggregated social presence to evaluate the average value obtained from the two selected sub-scales.

Regarding aggregated social presence, we found significantly higher scores on the solitary control (A) condition in the local space when compared to the MRC space $(Z=-4.114, p<0.0001)$ as shown in Figure 4.7. No statistical significance was noticed in the other two conditions, identical control (B) and limited control (C), between the two spaces. We 
Social presence

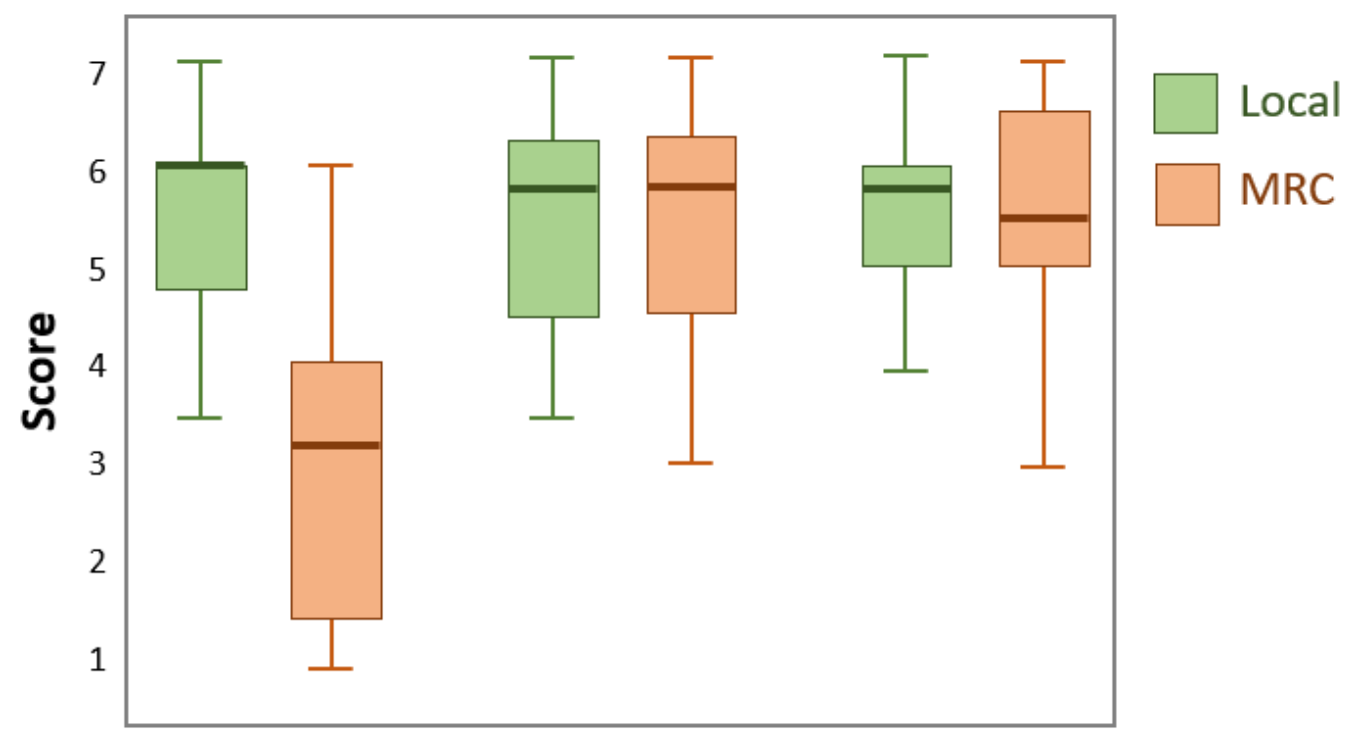

A: Solitary control B: Identical control C: Limited control

Figure 4.7: Social presence results between different control mechanisms. Green represents the local space, and orange represents the MRC space.

found statistical significance in the solitary control regarding social presence when compared to the neutral line $\left(\chi^{2}(2)=33.909, p<0.0001\right)$, which indicated higher scores for social presence in the local space $(Z=$ $-4.063, p<0.0001)$, and lower scores in the MRC space $(Z=-2.547, p=$ 0.011). However, we noted that identical control and limited control enabled significantly higher social presence scores in both spaces compared to the neutral line $\left(\mathrm{B}: \chi^{2}(2)=27.494, p<0.0001, \mathrm{C}: \chi^{2}(2)=26.068, p<\right.$ $0.0001)$, which indicated high scores for this metric. In the identical control condition, social presence was significantly better than at the neutral line both in the MRC space $(Z=-3.883, p<0.0001)$ and the local space $(Z=-4.006, p<0.0001)$. Limited control also resulted in significantly better social presence in both the MRC space $(Z=-3.878, p<0.0001)$ and the local space $(Z=-3.935, p<0.0001)$ when compared to the neutral line. 
When analysing the sub-scales, we found a significant difference in impression of interaction possibilities (IIP) sub-scale only in the solitary control. In the solitary control condition, participants were able to interact better with each other in the local space rather than the MRC space $(Z=-3.742, p<0.0001)$. We highlight that IIP in the local space scored higher than neutral line $(Z=-3.376, p=0.001)$, while the score was lower than neutral line in the MRC space $(Z=-2.204, p=0.028)$. On the other hand, no significant difference was found between the two spaces for identical and limited conditions. Even though there was no significant difference in IIP between the local space and the MRC space, both spaces enabled significantly better IIP than the neutral line (B: $\chi^{2}(2)=$ $\left.15.680, p<0.0001, \mathrm{C}: \chi^{2}(2)=22.627, p<0.0001\right)$. Lastly, the questions related to co-presence showed the same result as that reported in the IIP sub-scale, where solitary control allowed participants to feel a higher sense of being co-located in the local space rather than in the MRC space ( $Z=$ $-3.839, p<0.0001)$. Also, it indicated higher co-presence score in the local space $(Z=-3.895, p<0.0001)$, while the score was lower in the MRC space $(Z=-2.564, p=0.008)$. No statistical significance was found for the other two control conditions; however, both spaces enabled significantly better co-presence than the neutral line (B: $\chi^{2}(2)=32.107, p<0.0001, \mathrm{C}$ : $\left.\chi^{2}(2)=32.000, p<0.0001\right)$.

\section{User Preferences}

We used questionnaires to evaluate overall user preferences regarding both using a controller and the overall experience of interacting and collaborating with other participants. User preferences in different control mechanisms for collaboration were measured by task easiness, coordination easiness, and performance (Figure 4.8).

To check whether participants found the controller easy to use in performing the task, we measured the ease of manipulation against the neutral line. We found that our controller enabled higher scores when com- 


\begin{tabular}{c|l|l|l}
\hline \multirow{2}{*}{ Presence } & \multicolumn{3}{|c}{ Local space - MRC space } \\
\cline { 2 - 4 } & \multicolumn{1}{|c}{ Solitary $(\mathrm{A})$} & Identical $(\mathrm{B})$ & Limited $(\mathrm{C})$ \\
\hline Spatial presence & $\mathrm{Z}=-1.393, \mathrm{p}=0.164$ & $\mathrm{Z}=-2.400, \mathrm{p}=0.016^{*}$ & $\mathrm{Z}=-3.224, \mathrm{p}=0.001^{*}$ \\
AA & $\mathrm{Z}=-4.026, \mathrm{p}<0.0001^{*}$ & $\mathrm{Z}=-3.861, \mathrm{p}<0.0001^{*}$ & $\mathrm{Z}=-4.182, \mathrm{p}<0.0001^{*}$ \\
SSM & $\mathrm{Z}=-2.616, \mathrm{p}=0.009^{*}$ & $\mathrm{Z}=-2.622, \mathrm{p}=0.009^{*}$ & $\mathrm{Z}=-3.436, \mathrm{p}=0.001^{*}$ \\
SPSL & $\mathrm{Z}=-1.792, \mathrm{p}=0.073$ & $\mathrm{Z}=-1.664, \mathrm{p}=0.096$ & $\mathrm{Z}=-0.921, \mathrm{p}=0.357$ \\
SPPA & $\mathrm{Z}=-2.400, \mathrm{p}=0.016^{*}$ & $\mathrm{Z}=-0.068, \mathrm{p}=0.946$ & $\mathrm{Z}=-0.479, \mathrm{p}=0.632$ \\
\hline Social presence & $\mathrm{Z}=-4.114, \mathrm{p}<0.0001^{*}$ & $\mathrm{Z}=-0.032, \mathrm{p}=0.974$ & $\mathrm{Z}=-0.482, \mathrm{p}=0.630$ \\
IIP & $\mathrm{Z}=-3.742, \mathrm{p}<0.0001^{*}$ & $\mathrm{Z}=-0.288, \mathrm{p}=0.773$ & $\mathrm{Z}=-1.038, \mathrm{p}=0.299$ \\
Co-presence & $\mathrm{Z}=-3.839, \mathrm{p}<0.0001^{*}$ & $\mathrm{Z}=-0.832, \mathrm{p}=0.405$ & $\mathrm{Z}=-0.362, \mathrm{p}=0.717$ \\
\hline
\end{tabular}

Table 4.5: The presence result of Wilcoxon Signed Ranks Test $(a=0.05)$ as post-hoc tests between local space and MRC space in each control mechanism. Star $\left(^{*}\right)$ indicates statistical significance.

pared to the neutral line (It was easy to locate the objects: $Z=-4.516, p<$ 0.0001 , rotate: $Z=-2.175, p=0.030$, scale: $Z=-2.447, p=0.014$, and move the camera: $Z=-3.437, p=0.001)$.

We found statistical significance in the task easiness factor between different control mechanisms $\left(\chi^{2}(2)=7.275, p=0.026\right)$. In this matter, participants felt it was easier to perform the task with the limited control than solitary control $(Z=-2.363, p=0.018)$. We noticed that even though identical control $(Z=-3.214, p=0.001)$ and limited control ( $Z=-3.095, p=0.002$ ) were better than the neutral line for completing the task, no significant difference was found between solitary control and the neutral line.

There was also no significant difference in coordinating movements with other participants between conditions, but we found significant difference compared to the neutral line $\left(\chi^{2}(2)=22.962, p<0.0001\right)$. All the conditions enabled a better score than the neutral line (A: $Z=-2.131, p=$ 0.033, B: $Z=-3.296, p=0.001, \mathrm{C}: Z=-4.165, p<0.0001)$, indicating high scores. Participants felt it was easier to coordinate movements using the limited control condition ( $\mathrm{Md}=6, \mathrm{IQR}=1.75)$, followed by identical 


\begin{tabular}{|c|c|c|c|c|c|c|c|c|c|c|c|c|}
\hline \multirow{3}{*}{ Presence } & \multicolumn{3}{|c|}{ Local space } & \multicolumn{3}{|c|}{ MRC space } & \multicolumn{6}{|c|}{ Neutral } \\
\hline & \multirow[t]{2}{*}{$\begin{array}{c}\text { Solitary } \\
\text { (A) }\end{array}$} & \multirow[t]{2}{*}{$\begin{array}{l}\text { Identical } \\
\text { (B) }\end{array}$} & \multirow[t]{2}{*}{$\begin{array}{l}\text { Limited } \\
\text { (C) }\end{array}$} & \multirow[t]{2}{*}{$\begin{array}{l}\text { Solitary } \\
\text { (A) }\end{array}$} & \multirow[t]{2}{*}{$\begin{array}{c}\text { Identical } \\
\text { (B) }\end{array}$} & \multirow[t]{2}{*}{$\begin{array}{l}\text { Limited } \\
\text { (C) }\end{array}$} & \multicolumn{2}{|c|}{ Solitary(A) } & \multicolumn{2}{|c|}{ Identical (B) } & \multicolumn{2}{|c|}{ Limited (C) } \\
\hline & & & & & & & $\chi^{2}$ & p-value & $\chi^{2}$ & p-value & $\chi^{2}$ & p-value \\
\hline Spatial presence & 0.870 & 0.740 & 0.986 & 0.153 & $0.001^{*}$ & $<0.0001^{*}$ & 3.379 & 0.185 & 7.349 & 0.025 & 14.506 & $0.001^{*}$ \\
\hline AA & $<0.0001^{*}$ & 0.066 & $0.004^{*}$ & $<0.0001^{*}$ & $<0.0001^{*}$ & $<0.0001^{*}$ & 32.076 & $<0.0001^{*}$ & 29.585 & $<0.0001^{*}$ & 37.264 & $<0.0001^{*}$ \\
\hline SSM & 0.760 & 0.771 & 0.304 & $0.004^{*}$ & $0.001^{*}$ & $<0.0001^{*}$ & 10.800 & $0.005^{*}$ & 8.479 & $0.014^{*}$ & 20.182 & $<0.0001^{*}$ \\
\hline SPSL & 0.039 & 0.055 & 0.084 & 0.388 & 0.522 & 0.819 & 4.300 & 0.116 & 6.200 & 0.045 & 2.270 & 0.321 \\
\hline SPPA & 0.192 & 0.192 & 0.163 & 0.021 & 0.053 & 0.357 & 6.676 & 0.036 & 3.634 & 0.163 & 3.162 & 0.206 \\
\hline Social pre & $<0.0001^{*}$ & $<0.0001^{*}$ & $<0.0001^{*}$ & $0.011^{*}$ & $<0.0001^{*}$ & $<0.0001^{*}$ & \begin{tabular}{|l|}
33.909 \\
\end{tabular} & $<0.0001^{*}$ & 27.494 & $<0.0001^{*}$ & 26.068 & $<0.0001^{*}$ \\
\hline IIP & $0.001^{*}$ & $0.001^{*}$ & $<0.0001^{*}$ & $0.028^{*}$ & $0.002^{*}$ & $0.005^{*}$ & 24.317 & $<0.0001^{*}$ & 15.680 & $<0.0001^{*}$ & 22.627 & $<0.0001^{*}$ \\
\hline Co-presence & $<0.0001^{*}$ & $0.008^{*}$ & $<0.0001^{*}$ & $0.008^{*}$ & $<0.0001^{*}$ & $<0.0001^{*}$ & 31.063 & $<0.0001^{*}$ & 32.107 & $<0.0001^{*}$ & 32.000 & $<0.0001^{*}$ \\
\hline
\end{tabular}

Table 4.6: The presence result of Friedman parametric tests with Wilcoxon Signed Ranks Test $(a=0.05)$ as post-hoc tests between rating scores of presence and the neutral line in each control mechanism.

The Bonferroni correction (sig. = sig./3) was applied.

Star $\left(^{*}\right)$ indicates statistical significance.

control $(\mathrm{Md}=5.5, \mathrm{IQR}=1)$, and solitary control $(\mathrm{Md}=4.5, \mathrm{IQR}=2.75)$.

In terms of subjective opinions' about their own performance, we found statistical significance $\left(\chi^{2}(2)=18.083, p<0.0001\right)$. The results revealed that limited control was better than solitary control $(Z=-3.584, p<$ 0.0001 and identical control $(Z=-2.287, p=0.022)$. Identical control was also better than solitary control $(Z=-2.646, p=0.008)$. We found significant difference when comparing to neutral line $\left(\chi^{2}(2)=37.312, p<\right.$ $0.0001)$ in both identical control $(Z=-3.392, p=0.001)$ and limited control $(Z=-4.283, p<0.0001)$, while there was no significant difference between solitary control and the neutral line.

Lastly, participants felt that their performance was considerably affected by other participants' performance $\left(\chi^{2}(2)=12.522, p=0.002\right)$. Both identical control $(Z=-2.345, p=0.019)$ and limited control $(Z=$ $-2.705, p=0.007$ ) were significantly better than solitary control, while there was no significant difference between identical control and limited control. Similarly, even though these two conditions $\left(\chi^{2}(3)=26.444, p<\right.$ $0.0001)$ were significantly better than the neutral line (B: $Z=-3.494, p<$ $0.0001, \mathrm{C}: Z=-3.485, p<0.0001)$, solitary control was not significantly 


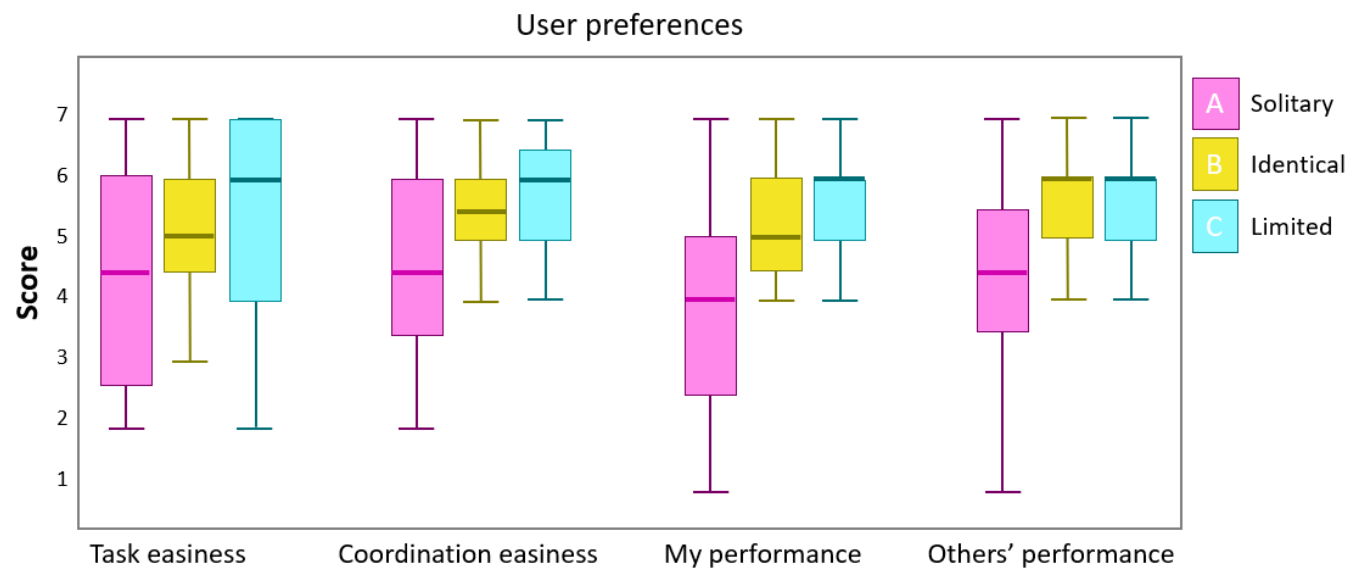

Figure 4.8: User preferences results analysed by four metrics.

Pink represents Solitary control (A), yellow represents Identical control (B), and sky blue represents Limited control (C).

different in comparison with the neutral line.

\subsubsection{Task Performance}

We gathered task performance measures through log files. The metrics selected for this were total task time, search time (time to find the target objects), manipulation time (time to rotate/scale the target objects), and precision (the difference between the required transform and transform of the target objects).

In terms of different control mechanisms, participants were slightly faster in completing the whole task under the limited control condition (C) $(\mathrm{Md}=245.3, \mathrm{IQR}=195.3)$ compared to solitary control $(\mathrm{A})(\mathrm{Md}=277.8$, $\mathrm{IQR}=264)$ and identical control $(\mathrm{B})(\mathrm{Md}=305.7, \mathrm{IQR}=207.4)$. However, there was no statistical significance in the total time measure (Figure 4.9).

Regarding the search time, we found a statistical significance in this metric $\left(\chi^{2}(2)=10.750, p=0.005\right)$. Pairwise comparisons showed that participants took less time to search for each target in the identical control condition when compared to the solitary control $(Z=-2.521, p=0.012)$. 


\begin{tabular}{l|c|c|c|c|c}
\hline \multirow{2}{*}{ User Preferences } & \multicolumn{3}{c|}{ Control mechanisms } & \multicolumn{2}{c}{} \\
\cline { 2 - 6 } & A - B & A - C & B - C & $\chi^{2}$ & p-value \\
\hline Task easiness & 0.062 & $0.018^{*}$ & 0.526 & 7.275 & $0.026^{*}$ \\
Coordination easiness & 0.158 & 0.011 & 0.186 & 3.455 & 0.178 \\
My performance & $0.008^{*}$ & $<0.0001^{*}$ & $0.022^{*}$ & 18.083 & $<0.0001^{*}$ \\
Others' performance & $0.019^{*}$ & $0.007^{*}$ & 0.726 & 12.522 & $0.002^{*}$ \\
\hline \multicolumn{7}{c}{ User Preferences } & Solitary (A) & Identical (B) & Limited (C) & \multicolumn{2}{c}{ Neutral } \\
\cline { 5 - 7 } & 0.522 & $0.001^{*}$ & $0.002^{*}$ & 16.360 & p-value \\
\hline Task easiness & $0.033^{*}$ & $0.001^{*}$ & $<0.0001^{*}$ & 22.962 & $<0.0001^{*}$ \\
Coordination easiness & 0.825 & $0.001^{*}$ & $<0.0001^{*}$ & 37.312 & $<0.0001^{*}$ \\
My performance & 0.364 & $<0.0001^{*}$ & $<0.0001^{*}$ & 26.444 & $<0.0001^{*}$ \\
Others' performance & \multicolumn{3}{|c}{}
\end{tabular}

Table 4.7: Top: (A) Solitary control, (B) Identical control, (c) Limited control. The user presences result of Wilcoxon Signed Ranks Test $(a=0.05)$ as post-hoc tests between different control mechanisms as a pairwise comparison. Bottom: The user presences result of Wilcoxon Signed Ranks Test $(a=0.05)$ as post-hoc tests between rating scores of user preferences and the neutral line in each control mechanism. Bonferroni correction (sig. = sig. /3) was applied. Star $\left(^{*}\right)$ indicates statistical significance.

We also found statistical significance in the rotation time $\left(\chi^{2}(2)=6.750, p=\right.$ $0.034)$ and scaling time $\left(\chi^{2}(2)=12.000, p=0.002\right)$. In this metric, we found that participants spent less time rotating objects in the identical control when compared to the solitary control $(Z=-1.960, p=0.050)$ and limited control $(Z=-2.380, p=0.017)$. Participants spent less time on scaling manipulations in the identical control when compared to solitary control $(Z=-2.521, p=0.012)$ and limited control $(Z=-2.521, p=0.012)$.

However, we found no statistically significant difference in precision. This is because we allowed participants to finish their manipulation task once they were satisfied with the arrangement. 


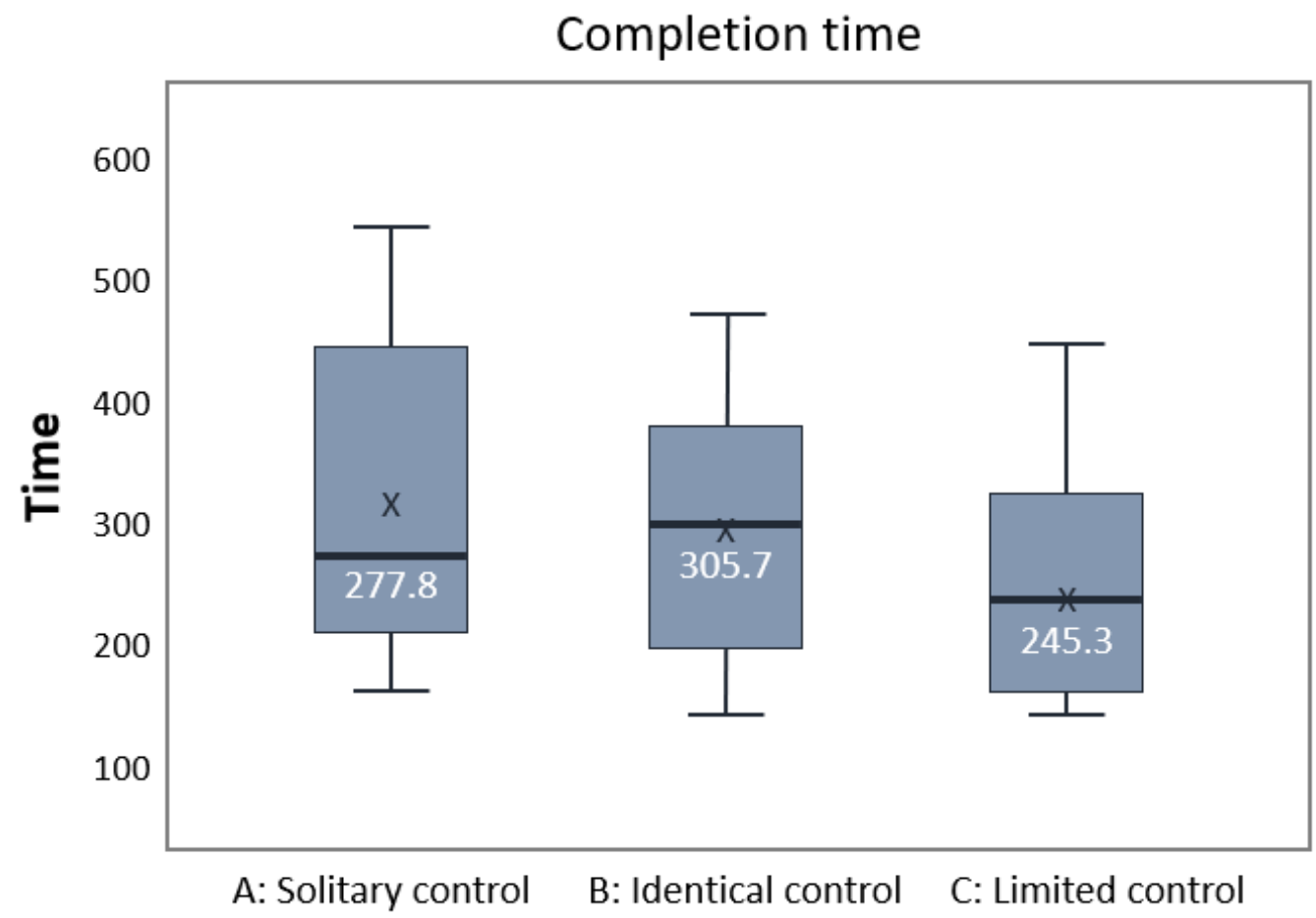

Figure 4.9: Boxplots representing the completion time per trial in seconds. 


\subsection{Chapter Summary}

In chapter 4, we evaluated our working prototype of TeleGate through a user study. Since TeleGate allows people to collaborate in the MRC space, while staying together in the same physical local space, our user study focused on evaluating presence in both spaces while collaborating. More specifically, we evaluated users' spatial and social presence and their preferences for collaboration when using our platform. We applied different control mechanisms by varying the three-levels of control to figure out how different control mechanisms can facilitate collaboration.

To answer our research questions, we designed a collaborative task regarding object manipulation around the large-scale displays. Two kinds of user interfaces were provided to support participants with controller manipulation and reference information. Participants performed collaborative tasks at the remote MRC space in a controlled environment.

To evaluate presence in both the MRC space and the local space, and user preferences for different control mechanisms, we deployed questionnaires associated with spatial presence, social presence, and user preferences to get subjective data. Objective data were also collected to analyse the task performance such as task, search, manipulation time, and precision. Lastly, we conducted parametric tests to draw statistically significant results from the data. The next chapter discusses how these results of the analysis can provide meaningful insight. 


\section{Chapter 5}

\section{Discussion}

In this chapter, we discuss what we discovered based on the analysis from the previous chapter. The first section contains our interpretation of spatial presence and social presence in both the MRC and the local space, exploring how different control mechanisms played a role in the presence. We also review user preferences and task performance influenced by different control mechanisms. We then discuss the feedback from participants, so as to improve our platform and suggest an application where our study can be adopted.

\subsection{Presence}

\subsubsection{Spatial Presence}

Our platform enabled participants to feel a higher sense of spatial presence in the MRC space rather than in their local space, confirming hypothesis H0. Notably, our setup allowed sub-scales (AA and SSM) of spatial presence to get higher scores in the MRC space in comparison to the neutral line, which consequently indicated a high sense of spatial presence.

We also found that the different control mechanisms played an important role when measuring the sense of spatial presence in each of the 
spaces. When all of the participants had some level of control, in both the identical and limited control conditions, spatial presence in the MRC space was higher than the neutral line, while no significant difference was found between the local space and the neutral line. This suggests that participants had a higher sense of spatial presence in the MRC space than in the local space.

We also noticed that the limited control condition supported participants in focusing their attention more in the MRC space than the local space. Participants stated that the limited control condition helped them to concentrate on their specific role when interacting in the MRC space. One of the participants even mentioned: "I could concentrate better on what I was doing since I was the only person who could perform camera movement" (P1).

In contrast, solitary control was found to limit some participants from being active in the MRC space, which resulted in a higher level of spatial presence in the local space compared to the MRC space. This is because two participants who participated only verbally in a group rated lower spatial presence in the MRC space because they collaborated more in the local space. Not having any controller brought them close as spectators who could only affect the MRC space indirectly. As indicated in the result, we can say that every user needs to wield influence in the MRC space to feel a better spatial presence in there.

\subsubsection{Social Presence}

Regarding social presence, our platform facilitated a high sense of social presence in both spaces when compared with the neutral line. We noted that the different control mechanisms also influenced social presence. When every participant had control over the environment (in both limited and identical control conditions), they maintained a similar level of social presence in both the local and MRC space. Since all participants 
could communicate with others in their local space and manipulate directly in the MRC space, their social presence in both spaces was considerably high. It was indicated by the result against the neutral line, confirming hypothesis $\mathrm{H} 1$.

However, when participants did not have any control over the scene in the solitary control condition, their level of social presence in the MRC space decreased considerably. Solitary control elicited a high sense of presence in the local space in comparison with the MRC space. In this case, social presence score in the MRC space was statistically lower than the neutral line, since participants' interaction was more active in their local space as a communication space. This can be explained by the need to describe verbally in detail to discuss their manipulation intentions.

Even though we could not find a statistically significant difference between identical and limited control, our observations from participants performing the tasks provided useful insights into how the different control mechanisms shaped the collaboration scenario. Identical control promoted the most active communication during the test session, as participants talked with and encouraged each other about what to do, which made their experience more enjoyable. The interview quotes below from two participants discussing their experience of the task under the identical control condition illustrate this: "I felt like we played as a team. Everyone affected the game performance" (P2); "It was so much fun for everyone to help each other, and we worked well as a group" (P3).

In contrast, limited control resulted in relatively less communication than the identical control, since every participant knew how they needed to collaborate to accomplish the task, without the need to discuss their role. Still, although participants needed to communicate less in this condition, the limited control mechanism encouraged participants to be more engaged, as they said: "I was more involved in the game in this condition" (P4); "Having each person doing a separate task is a great idea. I felt a lot more engaged" (P5). 


\subsection{User preferences}

According to our results from the questionnaire data analysis, participants found it easy to accomplish the set tasks. In terms of how the control mechanisms benefited collaboration, it can be said that participants overall preferred the condition where they had clear roles in collaboration, which was realised in our study through the limited control condition. This is backed up by participants' statements in the post-test interview, where there was a strong preference for the limited control condition (18 people or $75 \%$ of the participants), while only 6 people preferred the identical control condition, thus confirming hypothesis $\mathrm{H} 2$. We also noticed that participants established a collaboration protocol by themselves. In most cases, one participant controlled the camera, and the others performed object selection and manipulation. One of the eight participating groups split their roles even in the identical control condition, just like in the limited control condition. This was a deliberate choice by the participants in that group and not preset by the conditions order of our study design (limited control was the first condition performed by participants).

Regarding task easiness, our results show that participants preferred limited control to the solitary control condition. From the post-interview data, it was evident that limited control was seen as the easiest condition when compared to the other conditions. Participants stated that limited condition facilitated collaboration, as they were assigned clear roles to achieve their common goal. They mentioned: "I believe this way is the easiest one. Everything went very smoothly" (P6); "It is easier to cooperate when we are assigned to do something specific" (P7); "It was easier when we all had separate tasks to divide it out (P8)". We could not find a significant difference between identical control and limited control; however, we observed that some of the participants got confused with the identical control condition since they had the same control as others, stating for example: "All got confused and tried to focus on different things" (P9). 
In terms of coordinating movements with other participants, we could not find a statistical difference between different control mechanisms. Every control mechanism allowed participants to feel that they could easily express their opinions and coordinate their actions well on the task with others, as shown by the results. With this result, we can state that participants were able to communicate well to achieve the task regardless of the condition. Even if a collapse happened during the task with identical control, participants could discuss well in the communication space on how to proceed to overcome it. Particularly, in the case of the identical control condition, we observed that $50 \%$ of the groups established a collaboration protocol while doing the task. Interestingly enough, participants that did not establish a collaboration protocol performed the task slightly faster, as they could focus simultaneously on separate parts of the MRC space and position different objects at the same time.

Regarding performance, when asked about their contribution to other participants' performance, participants most expressed a high sense of contribution to other participants' performance with limited control, followed by the identical type. Participants with limited control were confident of their performance, indicating: "I had a sense that I could contribute to the completion of the task" (P10); "My performance definitely affected the other two participants" (P11). On the other hand, solitary control caused participants to feel that they could not make meaningful contributions. For example, one participant stated: "I just felt like a passive observer" (P12).

However, when evaluating other participants' contribution to their performance, there was no significant difference between identical control and limited control. Thus, we argue that granting all participants the same level of control is not desirable for co-located collaboration. 


\subsection{Task Performance}

We found no statistically significant difference in the completion time between the various control mechanisms. Because task performance is affected by various factors such as individual capabilities, the time varied depending on who participated and how the collaboration was realised. For instance, identical control recorded the highest median for the completion time, due to some groups of participants who actively discussed their idea during the task. In the same manner, the completion time in solitary control was easily affected by one's ability to manipulate.

However, we found that collaboration with identical control enabled better object manipulation. Identical control provided less time to find the target objects than solitary control and fastest rotation and scaling performance. From our observation, this is because identical control allowed more experienced participants to interfere and help with the task at any time.

\subsection{Suggested System Improvements}

Three aspects can be improved based on the suggested feedback from the participants: controller, camera perspective, and user interface.

- Controller: The controller was considered to be useful in general. However, it took some time to get used to it, since the controller came with up to four buttons. After the training session involving several trials, participants could adjust well to using the controller. Several participants commented: "The controller was hard to use at first, but when I eventually got used to the controls, it got easier to move around (P13, P14, P15)". However, some participants pointed out that putting the object farther or closer from them was a bit of a struggle, as they had to push or pull the controller to achieve this. 
- Camera perspective: The projection of 360-videos from Unity engine to the large-scale displays required the scene to be stretched to fit into displays' ratio; the distortion occurred on the edge of the displays. One of the participants pointed out that this inconsistent projection interrupted them from doing the task. It could distract participants from looking for objects located on the side; however, this can be resolved by rotating the camera to relocate the objects at the centre.

- User Interface: Some participants suggested that some interfaces are needed to direct them where to rotate the camera to manage the perspective easier. They also suggested that it would be better if they were given clear indicators which turn up when the right rotation and scale of the target objects have been achieved.

\subsection{Application}

In summary, we can state that participants preferred the conditions where every user could act in the remote MRC space. Both identical and limited control conditions enabled them to feel better spatial presence and social presence in the MRC space, while solitary control condition led participants to focus more on their local space.

The task was altogether easier to accomplish in the condition where multiple users had individual controllers, compared to when only one user had control. However, when asked to rank their favourite control mechanisms, most participants preferred the limited control condition because of the pre-established collaboration protocol, followed by identical control. This suggests that having a different degree of individual control when contributing to a collaborative task makes users feel more engaged compared to the same degree of control condition.

Therefore, when considering platform requirements using the same platform for collaborators to share, such as large-scale displays, granting 
all users the same level of control is not essential for effective collaboration. We argue that when co-located multiple users need to semi-teleport to affect the remote MRC space, a proper control mechanism needs to be designed to promote better user satisfaction. When a collaborative work is task-oriented, we suggest assigning each user a different role, with the ability to support one another. In contrast, when collaborative work requires active discussion between users, giving the same level of control would be appropriate.

Lastly, we suggest that our system is beneficial in scenarios that involve multiple experts in the same place having a face-to-face discussion while collaborating in the remote space without losing time for travelling. Our system can be leveraged for a live concert, allowing many spectators to interactively participate as though they were semi-teleporting simultaneously to a distant place.

\subsection{Chapter Summary}

In this chapter 5, we confirmed our research hypotheses formulated from the discussion. Our platform enabled participants to feel a higher sense of spatial presence in the MRC space rather than in their local space, which confirmed hypothesis H0. In addition to this, it facilitated a high sense of social presence in both spaces, which confirmed hypothesis H1. We also identified that participants could accomplish the collaborative tasks easily using TeleGate and they preferred to have some control over the environment, which confirmed hypothesis H2. Finally, we came up with some suggestions from participants for improving our platform. Based on this discussion, we have proposed an appropriate control mechanism to promote effective co-located collaboration and potential usage of our platform. Chapter 6 collates and summarises our discussion to conclude our work. 


\section{Chapter 6}

\section{Conclusion}

We presented "TeleGate", a solution for multi-user teleportation in MR space. TeleGate enables multiple users to semi-teleport to a distant location, allowing them to collaborate in the remote workspace while staying in their local physical space. Therefore, teleporters who are located in the same physical space can communicate naturally with each other in their local space while having a spatial presence in the remote collaboration space.

We implemented our platform using the large-scale displays as a portal that bridges the physical communication space and the remote collaboration space. The MRC space was implemented using 360-videos with seamlessly blended virtual objects. The users share the view of the remote MRC space through the large-scale displays performing collaborative tasks by manipulating virtual objects in the MRC space.

To evaluate our concept of semi-teleportation, we measured the spatial presence and social presence during collaboration by a user study. The user study also evaluated different collaboration strategies to figure out user preferences and suggest proper control mechanisms. We tested this collaborative task for object manipulation by varying a level of control: where only one user had control over the environment (A); where all users had the same control over the environment at the same time (B); where all 
users had control over the environment by having a specific role (C).

Our results showed that multiple users felt a higher spatial presence in the MRC space than their local physical space when they actively controlled elements in the environment. The social presence was also influenced by the level of control, with users with some control over the environment being able to maintain high levels of social presence in both spaces, enabling semi-teleportation. In conditions with no control, users were not able to teleport as they only indicated a high sense of social presence in their local space and low spatial presence in the MRC space. Users also found our platform effective to conduct the collaborative task and preferred the collaboration where everyone was assigned the specific role to influence the remote workspace together.

In the following section, we summarise the limitations of our work and discuss future research directions.

\subsection{Limitation and Future Work}

Regarding implementation, even though our platform can provide high fidelity of remote surrounding environment using 360-camera, there are still approximately two seconds of latency while streaming data from 360camera to local space. Therefore, in our user study, we used the prerecorded 360-video instead of using live streaming to minimise latency caused by networking. However, our network protocol needs to be improved to reduce latency for promoting better presence and collaboration when it comes to applying our platform to real-time scenarios. We expect that the emergence of the fifth generation (5G) of wireless technology will also bring minimal latency and possibility to connect through great distance better.

Due to 360-camera mobility limitations, we blocked an opportunity for users to navigate the MRC space. However, allowing users to explore the MRC space as if they are moving inside the environment could potentially 
increase users' sense of feeling immersed [70]; thus we can explore an option to adapt a robot where 360-camera is mounted for mobility.

In addition, our current platform only enables co-located users to collaborate while the remote side of the interaction contains the environment without any remote users. Our platform can be expanded in future work to put a remote user in the MRC space and enable multiple local users and the remote user to communicate with each other by adding audio communication cues. Then, comparative user evaluation can be conducted to investigate if the embodiment of the remote user can increase the presence compared to having no one in the MRC space. However, it is an open research question about how to present multiple users for the remote users who do not leverage the large-scale displays in this case. We assume that enabling group-to-group collaboration with contextual information using 360-video on both sides can potentially lead to enhanced presence and collaboration.

In terms of user study, participants were recruited as a group under the premise that they knew each other. A different result may have eventuated if people did not know each other. In future work, we will further explore providing a new method to measure the spatial presence and social presence specialising the concept of semi-teleportation based on our proposed platform. 


\subsection{Chapter Summary}

In conclusion, TeleGate enabled multiple co-located users to semi-teleport together into a remote space using large-scale displays, overcoming previous limitations of CVEs. Our approach maintains a natural communication between multiple users to conduct the collaborative work interacting with 360-video filmed from remote space. From our user study, we confirmed that multiple users were able to semi-teleport using our platform feeling a high sense of spatial presence in a remote environment and a high sense of social presence in their local space as well. Even though TeleGate has a limitation in latency streaming data from 360-camera, we will explore further to minimise latency in future work. Also, we will expand our work by putting remote users in the MRC space to support two-way communication for better teleportation and collaboration. We expect that our platform will open up huge possibilities for various industries which require multiple experts in the same place to have a face-to-face discussion or many spectators to interactively participate in any remote space in real time as though they have semi-teleported to a distant place altogether. 


\section{Bibliography}

[1] Adcock, M., Anderson, S., And Thomas, B. Remotefusion: Real time depth camera fusion for remote collaboration on physical tasks. In Proceedings of the 12th ACM SIGGRAPH International Conference on Virtual-Reality Continuum and Its Applications in Industry (New York, NY, USA, 2013), VRCAI '13, Association for Computing Machinery, p. 235-242.

[2] Aggarwal, A. Hoppin' world: The first social vr platform that allows for multi-user immersive remote teleportation using $360^{\circ}$ videos. https: / /hoppin.world.

[3] Argelaguet, F., Kunert, A., KuliK, A., And Froehlich, B. Improving co-located collaboration with show-through techniques. In Proceedings of the 2010 IEEE Symposium on 3D User Interfaces (USA, 2010), 3DUI '10, IEEE Computer Society, p. 55-62.

[4] Argyle, M., CoOK, M., And Cramer, D. Gaze and mutual gaze. British Journal of Psychiatry 165, 6 (1994), 848-850.

[5] Beck, S., Kunert, A., KuliK, A., And FrOeHlich, B. Immersive group-to-group telepresence. IEEE Transactions on Visualization and Computer Graphics 19, 4 (2013), 616-625.

[6] Beck, S., Kunert, A., KuliK, A., And Froehlich, B. Immersive group-to-group telepresence. IEEE Transactions on Visualization and Computer Graphics 19, 4 (2013), 616-625. 
[7] Benford, S., Bowers, J., FAHLÉN, L. E., MARIANi, J., AND RodDEN, T. Supporting Cooperative Work in Virtual Environments. The Computer Journal 37, 8 (01 1994), 653-668.

[8] BeNKO, H., ISHAK, E. W., AND FEINER, S. Collaborative mixed reality visualization of an archaeological excavation. In Proceedings of the 3rd IEEE/ACM International Symposium on Mixed and Augmented Reality (USA, 2004), ISMAR '04, IEEE Computer Society, p. 132-140.

[9] Billinghurst, M., AND KATO, H. Collaborative augmented reality. Commun. ACM 45, 7 (July 2002), 64-70.

[10] Billinghurst, M., Kato, H., KiyokaWA, K., Belcher, D., AND POUPYREV, I. Experiments with face-to-face collaborative ar interfaces. Virtual Reality 6 (10 2002), 107-121.

[11] Billinghurst, M., Weghorst, S., And Furness, T. A. Shared space: An augmented reality approach for computer supported collaborative work. Virtual Reality 3 (1998), 25-36.

[12] BiocCA, F. The Cyborg's Dilemma: Progressive Embodiment in Virtual Environments [1]. Journal of Computer-Mediated Communication 3, 2 (09 1997). JCMC324.

[13] BIOCCA, F., HARMS, C., AND BuRGOON, J. K. Toward a more robust theory and measure of social presence: Review and suggested criteria. Presence: Teleoperators and Virtual Environments 12, 5 (oct 2003), 456-480.

[14] BioccA, F., HARMs, C., AND GREGG, J. The networked minds measure of social presence: Pilot test of the factor structure and concurrent validity. 4th annual International Workshop on Presence, Philadelphia (01 2001). 
[15] Bowman, D. A., BADIllo, B., AND MANEK, D. Evaluating the need for display-specific and device-specific 3D interaction techniques. In Virtual Reality (Berlin, Heidelberg, 2007), R. Shumaker, Ed., Springer Berlin Heidelberg, pp. 195-204.

[16] Bowman, D. A., Johnson, D. B., AND Hodges, L. F. Testbed evaluation of virtual environment interaction techniques. In Proceedings of the ACM Symposium on Virtual Reality Software and Technology (New York, NY, USA, 1999), VRST '99, Association for Computing Machinery, p. 26-33.

[17] Churchill, E., AND SNOWDON, D. Collaborative virtual environments: An introductory review of issues and systems. Virtual Reality 3 (03 1998), 3-15.

[18] Churchill, E. F., AND SNOWdon, D. Collaborative virtual environments: An introductory review of issues and systems. Virtual Real. 3, 1 (Mar. 1998), 3-15.

[19] Cosgrove, S., AND LaViola, J. J. Visual guidance methods in immersive and interactive vr environments with connected $360^{\circ}$ videos. In 2020 IEEE Conference on Virtual Reality and 3D User Interfaces Abstracts and Workshops (VRW) (2020), pp. 653-654.

[20] Cruz-Neira, C., Sandin, D. J., DeFanti, T. A., Kenyon, R. V., AND HART, J. C. The cave: Audio visual experience automatic virtual environment. Commun. ACM 35, 6 (June 1992), 64-72.

[21] [de Klerk], R., Duarte, A. M., Medeiros, D. P., Duarte, J. P., JORGE, J., AND LOPES, D. S. Usability studies on building early stage architectural models in virtual reality. Automation in Construction 103 (2019), $104-116$.

[22] Febretti, A., Nishimoto, A., Thigpen, T., Talandis, J., Long, L., Pirtle, J. D., Peterka, T., Verlo, A., Brown, M., Plepys, D., 
SANDiN, D., ReNAmbOT, L., JOHNSON, A., AND LeIGH, J. CAVE2: a hybrid reality environment for immersive simulation and information analysis. In The Engineering Reality of Virtual Reality 2013 (2013), M. Dolinsky and I. E. McDowall, Eds., vol. 8649, International Society for Optics and Photonics, SPIE, pp. $9-20$.

[23] Feng ZhOu, DuH, H. B., And Billinghurst, M. Trends in augmented reality tracking, interaction and display: A review of ten years of ismar. In 2008 7th IEEE/ACM International Symposium on Mixed and Augmented Reality (2008), pp. 193-202.

[24] Fontaine, G. The experience of a sense of presence in intercultural and international encounters. Presence: Teleoper. Virtual Environ. 1, 4 (Jan. 1992), 482-490.

[25] FuCHS, H., STATE, A., AND BAZIN, J.-C. Immersive 3d telepresence. Computer 47, 7 (July 2014), 46-52.

[26] Fussell, S. R., KRAut, R. E., AND Siegel, J. Coordination of communication: Effects of shared visual context on collaborative work. In Proceedings of the 2000 ACM Conference on Computer Supported Cooperative Work (New York, NY, USA, 2000), CSCW '00, Association for Computing Machinery, p. 21-30.

[27] Fussell, S. R., KRAut, R. E., AND Siegel, J. Coordination of communication: Effects of shared visual context on collaborative work. In Proceedings of the 2000 ACM Conference on Computer Supported Cooperative Work (New York, NY, USA, 2000), CSCW '00, Association for Computing Machinery, p. 21-30.

[28] Gerathewohl, S. J. Fidelity of simulation and transfer of training: a review of the problem. Tech. rep., Civil Aerospace Medical Institute, 1969. 
[29] HartmanN, T., Wirth, W., Vorderer, P., Klimmt, C., SCHRAMm, H., AND BÖCKING, S. Spatial Presence Theory: State of the Art and Challenges Ahead. Springer International Publishing, Cham, 2015, pp. 115-135.

[30] Heeter, C. Being there: The subjective experience of presence. Presence: Teleoper. Virtual Environ. 1, 2 (May 1992), 262-271.

[31] Kim, S., LeE, G., SAKATA, N., AND Billinghurst, M. Improving co-presence with augmented visual communication cues for sharing experience through video conference. In 2014 IEEE International Symposium on Mixed and Augmented Reality (ISMAR) (2014), pp. 83-92.

[32] Kraut, R. E., Gergle, D., And Fussell, S. R. The use of visual information in shared visual spaces: Informing the development of virtual co-presence. In Proceedings of the 2002 ACM Conference on Computer Supported Cooperative Work (New York, NY, USA, 2002), CSCW '02, Association for Computing Machinery, p. 31-40.

[33] Kulik, A., Kunert, A., BecK, S., Reichel, R., Blach, R., ZinK, A., AND Froehlich, B. C1x6: A stereoscopic six-user display for co-located collaboration in shared virtual environments. ACM Trans. Graph. 30, 6 (Dec. 2011), 1-12.

[34] Kunert, A., Kulik, A., Beck, S., And Froehlich, B. Photoportals: Shared references in space and time. In Proceedings of the 17th ACM Conference on Computer Supported Cooperative Work \& Social Computing (New York, NY, USA, 2014), CSCW '14, Association for Computing Machinery, p. 1388-1399.

[35] Kunert, A., KuliK, A., Lux, C., And FröHlich, B. Facilitating system control in ray-based interaction tasks. In Proceedings of the 16th ACM Symposium on Virtual Reality Software and Technology (New 
York, NY, USA, 2009), VRST '09, Association for Computing Machinery, p. 183-186.

[36] Lacoche, J., Pallamin, N., Boggini, T., And Royan, J. Collaborators awareness for user cohabitation in co-located collaborative virtual environments. In Proceedings of the 23rd ACM Symposium on Virtual Reality Software and Technology (New York, NY, USA, 2017), VRST '17, Association for Computing Machinery.

[37] LANIER, J. Virtually there. Scientific American 284, 4 (2001), 66-75.

[38] LaViola, J., KruijfF, E., McMahan, R., Bowman, D., AND POUPYREV, I. 3D User Interfaces: Theory and Practice. Addison-Wesley usability and HCI series. Addison-Wesley, 2017.

[39] LEE, H., HA, G., LEE, S., AND KIM, S. A mixed reality tele-presence platform to exchange emotion and sensory information based on mpeg-v standard. In Virtual Reality (VR), 2017 IEEE (2017), IEEE, pp. 349-350.

[40] LEE, K. M. Presence, explicated. Communication Theory 14, 1 (01 2006), 27-50.

[41] López-IbÁÑez, M., ANd Peinado, F. Walking in vr: Measuring presence and simulator sickness in first-person virtual reality games. In CoSECivi (2016).

[42] Meehan, M., Insko, B., Whitton, M., And Brooks, JR, F. Physiological measures of presence in stressful virtual environments. ACM Transactions on Graphics 21 (08 2002).

[43] Mendes, D., Sousa, M., Araujo, B., Ferreira, A., Noronha, H., CAmpos, P., SoAres, L., Raposo, A., And Jorge, J. Collaborative $3 \mathrm{~d}$ visualization on large screen displays. In Powerwallinternational workshop on interactive, ultra-high-resolution displays-ACM CHI (2013), vol. 2013. 
[44] OH, C. S., BAILenson, J. N., AND Welch, G. F. A systematic review of social presence: Definition, antecedents, and implications. Frontiers in Robotics and AI 5 (2018), 114.

[45] Patrick, E., Cosgrove, D., Slavkovic, A., Rode, J. A., VerRATTI, T., AND CHISELKO, G. Using a large projection screen as an alternative to head-mounted displays for virtual environments. In Proceedings of the SIGCHI Conference on Human Factors in Computing Systems (New York, NY, USA, 2000), CHI '00, ACM, pp. 478-485.

[46] Patrick, E., Cosgrove, D., Slavkovic, A., Rode, J. A., VerRATTI, T., AND CHISELKO, G. Using a large projection screen as an alternative to head-mounted displays for virtual environments. In Proceedings of the SIGCHI Conference on Human Factors in Computing Systems (New York, NY, USA, 2000), CHI '00, ACM, pp. 478-485.

[47] Piumsomboon, T., Lee, G., Hart, J., Ens, B., Lindeman, R., ThOMAS, B., AND BILlinghuRst, M. Mini-me: an adaptive avatar for mixed reality remote collaboration. In CHI 2018 - Proceedings of the 2018 CHI Conference on Human Factors in Computing Systems (United States of America, 2018), A. Cox and M. Perry, Eds., Association for Computing Machinery (ACM).

[48] Piumsomboon, T., Lee, Y., Lee, G., And Billinghurst, M. Covar: a collaborative virtual and augmented reality system for remote collaboration. In 2017 International Conference on Computer Graphics and Interactive Techniques (11 2017), pp. 1-2.

[49] Poeschl-Guenther, S., AND Doering, N. Measuring co-presence and social presence in virtual environments - psychometric construction of a german scale for a fear of public speaking scenario. Annual Review of CyberTherapy and Telemedicine 13 (01 2015), 58-63. 
[50] Prothero, J., And Hoffman, H. Widening the field-of-view increases the sense of presence in immersive virtual environments.

[51] Prouzeau, A., Bezerianos, A., And Chapuis, O. Visual immersion in the context of wall displays. In Proceedings of the 2016 ACM Companion on Interactive Surfaces and Spaces (New York, NY, USA, 2016), ISS '16 Companion, Association for Computing Machinery, p. 33-39.

[52] Rhee, T., Petikam, L., Allen, B., And Chalmers, A. Mr360: Mixed reality rendering for $360^{\circ}$ panoramic videos. IEEE Transactions on Visualization and Computer Graphics 23, 4 (2017), 1379-1388.

[53] Rhee, T., Thompson, S., Medeiros, D., Anjos, R., AND Chalmers, A. Augmented virtual teleportation for high-fidelity telecollaboration. IEEE Transactions on Visualization and Computer Graphics PP (02 2020), 1-1.

[54] Sharples, S., CobB, S., Moody, A., And Wilson, J. R. Virtual reality induced symptoms and effects (VRISE): Comparison of head mounted display (HMD), desktop and projection display systems. Displays 29, 2 (2008), 58 - 69. Health and Safety Aspects of Visual Displays.

[55] SHERIDAN, T. B. Musings on telepresence and virtual presence. Presence: Teleoperators and Virtual Environments 1, 1 (1992), 120-126.

[56] Short, J., Williams, E., And Christie, B. The Social Psychology of Telecommunications. London: John Wiley \& Sons, 1976.

[57] SIMON, A. Usability of multiviewpoint images for spatial interaction in projection-based display systems. IEEE Transactions on Visualization and Computer Graphics 13, 1 (2007), 26-33. 
[58] Slater, M., McCarthy, J., And Maringelli, F. The influence of body movement on subjective presence in virtual environments. Human Factors 40, 3 (1998), 469-477. PMID: 9849105.

[59] Steuer, J. Defining virtual reality: Dimensions determining telepresence. Journal of Communication 42, 4 (02 2006), 73-93.

[60] Szalavári, Z., Schmalstieg, D., Fuhrmann, A., and GerVAUTZ, M. "studierstube": An environment for collaboration in augmented reality. Virtual Reality 3 (03 1998), 37-48.

[61] Tecchia, F., Alem, L., AND HuAnG, W. 3d helping hands: A gesture based $\mathrm{mr}$ system for remote collaboration. pp. 323-328.

[62] TeO, T., Lawrence, L., Lee, G. A., Billinghurst, M., AND ADCOCK, M. Mixed reality remote collaboration combining 360 video and 3d reconstruction. In Proceedings of the 2019 CHI Conference on Human Factors in Computing Systems (New York, NY, USA, 2019), CHI '19, ACM, pp. 201:1-201:14.

[63] Vorderer, P., Wirth, W., Gouveia, F., Biocca, F., SaAri, T., JÄNCKe, L., BÖCKING, S., SChramM, H., GYSBERS, A., HARTMANN, T., KLIMmt, C., LAARNi, J., RaVaja, N., SACAU, A., BAumGARTNER, T., AND JÄNCKE, P. A. Mec spatial presence questionnaire (mec-spq): Short documentation and instructions for application. Report to the European Community, Project Presence: MEC (IST-2001-37661) (06 2004).

[64] WARE, C., AND OSBORNE, S. Exploration and virtual camera control in virtual three dimensional environments. SIGGRAPH Comput. Graph. 24, 2 (Feb. 1990), 175-183.

[65] WARE, C., AND OSBORNE, S. Exploration and virtual camera control in virtual three dimensional environments. In Proceedings of the 1990 
Symposium on Interactive 3D Graphics (New York, NY, USA, 1990), I3D '90, Association for Computing Machinery, p. 175-183.

[66] Weech, S., Kenny, S., And Barnett-Cowan, M. Presence and cybersickness in virtual reality are negatively related: A review. Frontiers in Psychology 10 (2019), 158.

[67] Weibel, D., Schmutz, J., PAhud, O., And Wissmath, B. Measuring spatial presence: Introducing and validating the pictorial presence sam. Presence: Teleoperators and Virtual Environments 24, 1 (2015), 44-61.

[68] Welsford-Ackroyd, F., Chalmers, A., D. Anjos, R. K., MEdeIROS, D., KIM, H., AND RHEE, T. Asymmetric interaction between hmd wearers and spectators with a large display. In 2020 IEEE Conference on Virtual Reality and 3D User Interfaces Abstracts and Workshops (VRW) (2020), pp. 671-672.

[69] Wirth, W., Hartmann, T., BÖCKing, S., Vorderer, P., KLimmt, C., Schramm, H., SAari, T., LAarni, J., Ravaja, N., Gouveia, F., BIOCCA, F., SACAU, A., JÄNCKE, L., BAUMGARTNER, T., AND J ÄNCKE, P. A process model of the formation of spatial presence experiences. Media Psychology 9 (05 2007), 493-525.

[70] Witmer, B. G., AND Singer, M. J. Measuring presence in virtual environments: A presence questionnaire. Presence: Teleoperators and Virtual Environments 7, 3 (1998), 225-240.

[71] You, B.-J., KwON, J. R., NAM, S.-H., LEE, J.-J., LEE, K.-K., AND YEOM, K. Coexistent space: Toward seamless integration of real, virtual, and remote worlds for $4 \mathrm{~d}+$ interpersonal interaction and collaboration. In SIGGRAPH Asia 2014 Autonomous Virtual Humans and Social Robot for Telepresence (New York, NY, USA, 2014), SA '14, Association for Computing Machinery. 
[72] YoungBLUt, C. Experience of presence in virtual environments. 149. 